\title{
A finite strain incremental-secant homogenization model for elasto-plastic composites
}

\author{
M. I. El Ghezal ${ }^{\mathrm{a}, \mathrm{c}, *}$, L. Wu ${ }^{\mathrm{b}}$, L. Noels ${ }^{\mathrm{b}}$, I. Doghri ${ }^{\mathrm{a}}$ \\ ${ }^{a}$ Université catholique de Louvain (UCL), iMMC/MEMA, Bâtiment Euler, 4 Avenue G.Lemaître B-1348 \\ Louvain-La-Neuve, Belgium \\ ${ }^{b}$ Université de Liège, Department of Aeronautics and Mechanical Engineering, \\ Computational \& Multiscale Mechanics of Materials, Allée de la découverte 9, B-4000 Liège, Belgium \\ ${ }^{c}{ }^{e}$-Xstream Engineering, Axis Park-Building H, Rue Emile Francqui 9, B-1435 Mont-Saint-Guibert, \\ Belgium
}

\begin{abstract}
This paper presents a finite strain extension of the incremental-secant mean-field homogenization (MFH) formulation for two-phase elasto-plastic composites. The formulation of the local finite strain elasto-plastic constitutive equations of each phase is based on a multiplicative decomposition of the deformation gradient as suggested by Simo in (Computer Methods in Applied Mechanics and Engineering, 99(1):61-112, 1992.). The latter has proposed algorithms which preserve the classical return mapping schemes of the infinitesimal theory by using principal Kirchhoff stresses and logarithmic eigenvalues of the left elastic Cauchy-Green strain. Relying on this property, we show that, by considering a quadratic logarithmic free energy and J2-flow theory at the local level, infinitesimal strain incremental-secant MFH is readily extended to finite strains. The proposed formulation and corresponding numerical algorithms are then presented. Finally, the predictions are illustrated with several numerical simulations which are verified against full-field finite element simulations of composite cells, demonstrating that the micro-mechanically based approach is able to predict the influence of the micro-structure and of its evolution on the macroscopic properties in a very cost-effective manner.
\end{abstract}

\footnotetext{
*Corresponding author. Tel: +32-4-366 48 26. e-mail: 1.noels@ulg.ac.be

Email address: 1.noels@ulg. ac.be (L. Noels)
} 
Keywords: Mean-field homogenization, Incremental-secant formulation, Finite strains, Mori-Tanaka model, Composites

\section{Introduction}

The aim of scale transition methods is to understand and quantify the mutual interaction between the micro-structure and the effective (or "macroscopic") response. For composites, several scale transition methods exist which rely on a separation of scales and full-field or mean-field homogenization; e.g. see the reviews [1, 2]. Mean-field homogenization (MFH) is restricted to micro-structures such that multiple phases of solid inclusions or cavities, which are supposed to have either an ellipsoidal shape or whose spatial distribution follows an ellipsoidal symmetry in the sense of the spatial correlation [3], are embedded in a continuum matrix. MFH is based on assumed relations between the mean values (volume averages) of strain or stress fields in each phase and, unlike full-field methods, it is not direct, it does not solve for the detailed micro fields, and it is more restrictive than the latter both in terms of the micro-structures that can be handled and of the results that can be delivered. However, MFH is much easier to use and its computing time is several orders of magnitude smaller than that of direct scale-bridging methods, especially in the nonlinear regime.

MFH methods are based on the fundamental linear elastic solution developed in [5] for an ellipsoidal subdomain of an infinite matrix undergoing a uniform eigen or transformation strain. In linear elasticity, successful MFH models have been developed based on an approximate use of Eshelby's solution, for a review see [6]. A significant research effort has been devoted to extend MFH to nonlinear elasto(visco)plastic composites. In the small-deformation framework, the homogenization problem of the non-linear composite material is transformed into that of a Linear Comparison Composite (LCC) [7, 8, 9, 10, 11, 12]. Several linearization techniques were developed in order to define the LCC. Among them, the secant 


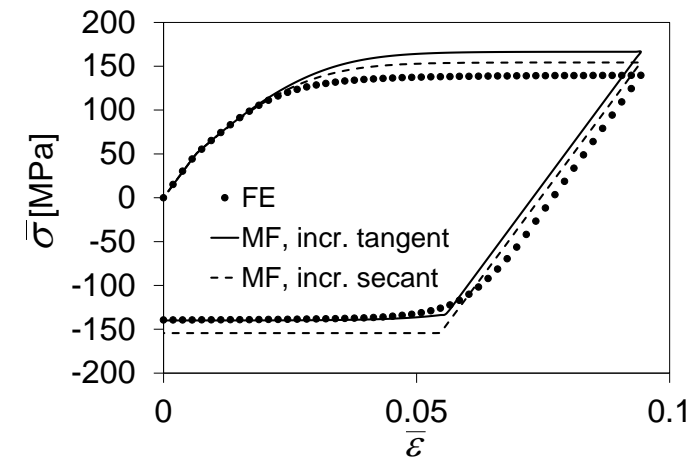

(a)

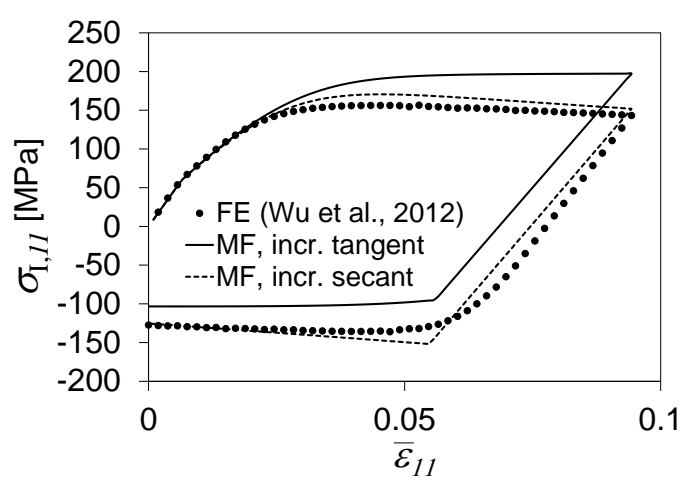

(b)

Figure 1: Stress evolution for elastic fibers embedded in a matrix obeying an elasto-plastic behavior with damage [4]. (a) Average stress in the composite material along the loading direction. (b) Average stress in the fibers phase along the loading direction.

formulation [13] determines for each phase a secant operator which links the total strain to the total stress. This method is limited to monotonic and proportional loading conditions. The incremental-tangent approaches are based on a rate formulation of the local problem $[14,15,16,17,18]$. The linearization is carried out by a discretization over each time interval via a tangent operator relating the stress and strain increments. Affine methods [19, 20, 21, 12, 22] also use tangent moduli and introduce a polarization stress. However, both the incremental-tangent and the affine methods need isotropic projections of the tangent operators in the homogenization process otherwise they yield too stiff estimates [23]. Moreover, the incremental tangent and affine formulations have some limitations in the case of non-proportional loading [24] or when considering a strain softening response [25]. In the latter case, $\mathrm{Wu}$ et al. [25] coupled a damage law in the constitutive model of the matrix phase for fiber-reinforced composites and showed that the incremental tangent formulation does not allow the fibers to unload during the softening stage of the matrix. These limitations have motivated the development of a new formulation [24]: the incremental-secant approach which, on the one hand, captures with high accuracy the composite material response during non proportional loading and, on the other hand, accounts, by design, for resulting unloading of the fibers during strain softening of the matrix [4]. Two main features 
distinguish the incremental-secant MFH scheme from the incremental tangent one. First, the residual stress and strain states in each phase are evaluated by applying a fictitious elastic unloading step. The mean stress fields in the phases are then computed using secant operators which are naturally isotropic, thus avoiding the isotropization step required by both the affine and incremental-tangent methods [23]. These secant operators are then used to define a Linear Comparison Composite (LCC) subjected to a strain increment deduced from the residual state. When damage in one phase is considered, the incremental-secant approach improves considerably the accuracy of the predictions compared to the incremental tangent approach. As explained in [4] and illustrated in Fig. 1-(b), the average stress along the loading direction in the fibers keeps increasing as predicted by the incremental-tangent model, however it should decrease after the damaging process in the matrix. This unloading is captured by the incremental-secant model which leads to much better predictions at the macroscopic and microscopic scales (see Fig. 1).

For recent reviews of the literature on the topic of non-linear MFH, we refer to [26] for infinitesimal strains, and [27] for finite transformation. In particular, MFH homogenization in finite strain has been developed based on a variational formulation for hyper-elastic composites [28, 29, 30] and porous materials [31].

An alternative MFH for finite transformations has been proposed in [27] in which the local phases follow a hyper-elastic-plastic material model built on the following formalism:

$$
\boldsymbol{F}=\boldsymbol{F}^{e} \cdot \boldsymbol{F}^{p}, \quad \boldsymbol{\tau}=2 \frac{\partial \psi}{\partial \boldsymbol{b}^{e}} \cdot \boldsymbol{b}^{e},
$$

i.e. a multiplicative decomposition of the deformation gradient $\boldsymbol{F}$ into elastic and inelastic parts, and a hyper-elastic stress strain relation between the Kirchhoff stress $\tau$ and the elastic left Cauchy-Green strain $\boldsymbol{b}^{e}=\boldsymbol{F}^{e} \cdot\left(\boldsymbol{F}^{e}\right)^{T}$, derived from a specific free energy per unit reference volume $\psi$. The formulation presents important advantages as compared to a more classical approach based on an additive decomposition of the rate of deformation tensor and on hypo-elasticity. Indeed, 
the former is not restricted to small elastic strains (which makes it suitable for polymers), the hyper-elastic stress-strain relation and the flow rule are derived from thermodynamic considerations, and there is no need to choose one objective stress rate over another (Jaumann, Green-Naghdi-Mc Innis, etc.). Besides, although the multiplicative decomposition $\boldsymbol{F}=\boldsymbol{F}^{e} \cdot \boldsymbol{F}^{p}$ initially proposed in [32] was successively used in computational mechanics without formal mathematical justification, it was shown in $[33,34]$ that such a decomposition allows writing the time continuous and time incremental formulations under the form of a variational incremental (or update) algorithm in which the stress tensor corresponds to the minimum of an effective potential itself solution of an extremum problem in terms of internal variables. The mathematical formalism of this variational incremental method was established in [35], yielding a global existence of rate-independent [36] and rate dependent [37] elasto-plasticity.

In the recently published paper [27], an incremental-tangent MFH formulation was proposed. Although it gives acceptable macroscopic predictions for most cases, the incremental tangent formulation has some limitations as discussed in the framework of small deformations here above, in particular in the case of nonproportional loading [24] or when considering a strain softening response [25]. For large deformations, ductile materials are much more likely to witness strain softening, i.e. a decrease in some stress measures with increasing strain. Therefore, as a step to a study of strain softening in large deformation, the goal of the present article is to construct a finite strain extension of the incremental-secant MFH scheme [24] initially formulated for infinitesimal strains. The micro-mechanical model and the corresponding numerical algorithm for two-phase elasto-plastic composites are presented. To this end, the local finite strain elasto-plasticity constitutive equations of each phase are based on a multiplicative decomposition of the deformation gradient as suggested by Simo [38]. In this work, we consider the specific case of a quadratic logarithmic free energy and J2-flow theory at the local level, which allows the incremental-secant model to be generalized from 
infinitesimal strains to finite strain elasto-plastic composites.

The paper is organized as follows. Sections 2 and 3 present some basic results related to, respectively, finite-deformation and hyper-elasto-plasticity needed to develop the MFH method. The incremental-secant MFH formulated in a finitedeformation setting is then developed in Section 4. Several numerical MFH predictions and their verification against direct FE simulations are presented and discussed in Section 5. Conclusions are drawn in Section 6.

\section{Kinematics of large deformations}

In this section, we summarize some basic results needed in the remainder of this paper. Throughout, boldface symbols designate tensors, the order of which is indicated by the context. Inner products over one and two indices are symbolized by a dot and a colon, respectively. The summation convention over repeated indices is used unless indicated otherwise. The tensor (dyadic) product is designated by the symbol $\otimes$.

A solid body, which in this paper designates a representative volume element (RVE) or a part of it, occupies a domain $\Omega_{0}$ (boundary $\partial \Omega_{0}$ ) in the reference configuration and a domain $\omega_{t}$ (boundary $\partial \omega_{t}$ ) in the current configuration at time

$t>0$. A fixed Cartesian frame is considered. A material particle is determined by its position vectors $X$ and $x$ with respect to (w.r.t.) $\Omega_{0}$ and $\omega_{t}$ so that for a motion $\varphi(X, t)$, we have:

$$
x=\varphi(X, t) ; \quad X=\left(X_{A}\right) ; \quad x=\left(x_{i}\right) ; \quad A, i=1,2,3 \text {. }
$$

The convention of upper case indices for $X_{A}$ and lower case for $x_{i}$ will be used throughout.

The deformation gradient and its determinant are defined as follows:

$$
\boldsymbol{F}(\boldsymbol{X}, t)=\frac{\partial x}{\partial X} \equiv \operatorname{GRAD} x, J(X, t)=\operatorname{det} \boldsymbol{F}(\boldsymbol{X}, t)
$$


From $\boldsymbol{F}$, the right $(\boldsymbol{C})$ and left $(\boldsymbol{b})$ Cauchy-Green strain tensors are computed:

$$
\boldsymbol{C}=\boldsymbol{F}^{T} \cdot \boldsymbol{F} ; \quad \boldsymbol{b}=\boldsymbol{F} \cdot \boldsymbol{F}^{T} .
$$

Both tensors are symmetric: $C_{A B}=C_{B A}$ and $b_{i j}=b_{j i}$.

Two stress measures are needed: the Cauchy (true) stress $\sigma=\left(\sigma_{i j}\right)$ and the Kirchhoff stress $\tau=\left(\tau_{i j}\right)=J \sigma$. The stress tensors $\sigma$ and $\tau$ are symmetric.

\section{Hyper-elastic-plastic constituents}

\subsection{Local constitutive equations}

At the local (micro) level, elasto(visco)plastic materials obey a finite strain formulation which is based on a multiplicative decomposition of the deformation gradient onto elastic and inelastic parts, and on hyper-elastic stress-strain response. The following summary follows [38]; see also Sect. 16.1 in [39] for instance.

The multiplicative decomposition of $\boldsymbol{F}$ into elastic $\left(\boldsymbol{F}^{e}\right)$ and inelastic $\left(\boldsymbol{F}^{p}\right)$ parts reads (Fig. 2 presents a schematic illustration):

$$
\boldsymbol{F}(\boldsymbol{X}, t)=\boldsymbol{F}^{e}(\boldsymbol{X}, t) \cdot \boldsymbol{F}^{p}(\boldsymbol{X}, t) .
$$

The elastic left $\left(\boldsymbol{b}^{\boldsymbol{e}}\right)$ and plastic right $\left(\boldsymbol{C}^{p}\right)$ Cauchy-Green strain tensors are defined as follows:

$$
\boldsymbol{b}^{e}=\boldsymbol{F}^{e} \cdot \boldsymbol{F}^{e T} ; \boldsymbol{C}^{p}=\boldsymbol{F}^{p T} \cdot \boldsymbol{F}^{p} .
$$

It follows from the multiplicative decomposition (5) that:

$$
\boldsymbol{b}^{e}=\boldsymbol{F} \cdot \boldsymbol{C}^{p^{-1}} \cdot \boldsymbol{F}^{T} .
$$

In the remainder of the paper, local materials are supposed isotropic. The equations of state are obtained from the non negativity of the Clausius-Duhem inequal- 


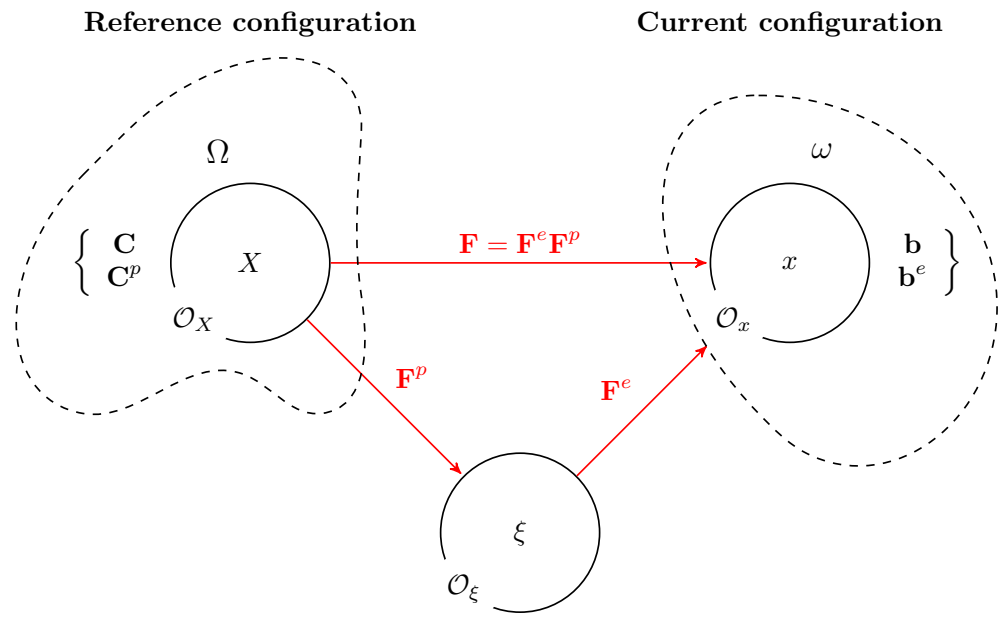

Stress-free intermediate configuration

Figure 2: The multiplicative decomposition of the deformation gradient: $\boldsymbol{F}^{e^{-1}}$ is interpreted as the local deformation that releases the stresses from each neighborhood $O_{x}$ in the current configuration. Strain tensors associated with the reference or current configurations are schematically indicated.

ity, as summarized in Appendix A, resulting in

$$
\boldsymbol{\tau}=2 \frac{\partial \psi}{\partial \boldsymbol{b}^{e}} \cdot \boldsymbol{b}^{e} ; q \equiv \frac{\partial \psi}{\partial \xi}
$$

where a specific free energy per unit reference volume $\psi\left(\boldsymbol{b}^{e}, \xi\right)$ is an isotropic function of $\boldsymbol{b}^{e}$ and of an internal scalar variable $\xi$. Equation (8-a) is a hyper-elastic relation between the Kirchhoff stress $\boldsymbol{\tau}$ and $\boldsymbol{b}^{e}$, while $q$ is the thermodynamic force associated with $\xi$ (typically, $q$ is a hardening stress). The reduced Clausius-Duhem dissipation inequality reads:

$$
\mathcal{D}=\boldsymbol{\tau}:\left(-\frac{1}{2} \boldsymbol{b}^{e} \cdot \boldsymbol{b}^{e-1}\right)-q \dot{\xi} \geq 0
$$

where $\boldsymbol{b}^{e} \equiv \boldsymbol{F} \cdot \frac{\mathrm{d}}{\mathrm{d} t}\left(\boldsymbol{C}^{p-1}\right) \cdot \boldsymbol{F}^{T}$ is the Lie derivative of $\boldsymbol{b}^{e}$ and is defined from the velocity gradient $\boldsymbol{l}=\dot{\boldsymbol{F}} \cdot \boldsymbol{F}^{-1}$ as :

$$
\stackrel{L}{\boldsymbol{b}^{e}}=\dot{\boldsymbol{b}}^{e}-\boldsymbol{l} \cdot \boldsymbol{b}^{e}-\boldsymbol{b}^{e} \cdot \boldsymbol{l}^{T}
$$


The flow rules are shown to be:

$$
-\frac{1}{2} \boldsymbol{b}^{e} \cdot \boldsymbol{b}^{e-1}=\dot{\gamma} \frac{\partial f}{\partial \tau}(\tau, q) ; \quad \dot{\xi}=-\dot{\gamma} \frac{\partial f}{\partial q}(\tau, q) ;
$$

where $f(\tau, q)$ is an isotropic yield function and $\dot{\gamma}$ is a consistency parameter. The left-hand-side of Eq. (11-a) plays the role of "plastic strain rate" tensor and can be rewritten as follows:

$$
-\frac{1}{2} \boldsymbol{b}^{L} \cdot \boldsymbol{b}^{e-1}=\frac{1}{2} \boldsymbol{F} \cdot \boldsymbol{C}^{p-1} \cdot \dot{\boldsymbol{C}}^{p} \cdot \boldsymbol{F}^{-1}
$$

For rate-independent hyper-elastic-plastic models, the following conditions hold:

$$
f(\tau, q) \leq 0 ; \quad \dot{\gamma} \geq 0 ; \quad \dot{\gamma} f(\tau, q)=0
$$

\subsection{Spectral decomposition}

The local material being isotropic, $\psi\left(\boldsymbol{b}^{e}, \xi\right)$ and $f(\tau, q)$ are isotropic functions

of $\boldsymbol{b}^{e}$ and $\tau$ respectively. Accordingly, $\boldsymbol{b}^{e}$ and $\frac{\partial \psi}{\partial \boldsymbol{b}^{e}}$ have the same eigenvectors and thus the principal directions of the Kirchhoff stress tensor $\tau$ and the elastic left Cauchy-Green tensor coincide.

$$
\boldsymbol{\tau}=\sum_{A=1}^{3} \tau_{A} \boldsymbol{n}_{t}^{(A)} \otimes \boldsymbol{n}_{t}^{(A)}, \quad \boldsymbol{b}^{e}=\sum_{A=1}^{3}\left(\lambda_{A}^{e}\right)^{2} \boldsymbol{n}_{t}^{(A)} \otimes \boldsymbol{n}_{t}^{(A)}
$$

where $\tau_{A}$ and $\left(\lambda_{A}^{e}\right)^{2}$ are the eigenvalues of $\boldsymbol{\tau}$ and $\boldsymbol{b}^{e}$ respectively. The restriction to isotropy also implies the existence of a function $\bar{\psi}$ such that $\psi\left(\boldsymbol{b}^{e}, \xi\right)=$ $\bar{\psi}\left(\left(\lambda_{1}^{e}\right)^{2},\left(\lambda_{2}^{e}\right)^{2},\left(\lambda_{3}^{e}\right)^{2}, \xi\right)$, a symmetric function of $\left(\lambda_{A}^{e}\right)^{2}$. Thus, using the stress strain Eq. (8-a), it is easily checked that $\tau_{A}$ is given by:

$$
\tau_{A}=2\left(\lambda_{A}^{e}\right)^{2} \frac{\partial \bar{\psi}}{\partial\left(\lambda_{A}^{e}\right)^{2}}
$$


The principal elastic logarithmic stretches are defined by the relation:

$$
\varepsilon_{A}^{e}=\ln \left(\lambda_{A}^{e}\right) .
$$

We introduce for convenience the following vector array notation:

$$
\underline{\boldsymbol{\tau}}=\left[\begin{array}{c}
\tau_{1} \\
\tau_{2} \\
\tau_{3}
\end{array}\right] ; \operatorname{dev}(\underline{\boldsymbol{\tau}})=\frac{1}{3}\left[\begin{array}{c}
2 \tau_{1}-\tau_{2}-\tau_{3} \\
2 \tau_{2}-\tau_{1}-\tau_{3} \\
2 \tau_{3}-\tau_{1}-\tau_{2}
\end{array}\right] ; \quad \underline{\varepsilon}^{e}=\left[\begin{array}{c}
\varepsilon_{1}^{e} \\
\varepsilon_{2}^{e} \\
\varepsilon_{3}^{e}
\end{array}\right] ; \quad \underline{\mathbf{1}}=\left[\begin{array}{c}
1 \\
1 \\
1
\end{array}\right] ; \quad \underline{\boldsymbol{I}}=\operatorname{diag}(1,1,1) ;
$$

where the notation $\operatorname{dev}(\underline{\tau})$ denotes the eigenvalues of the deviator of $\tau$. We also have the norm $\|\operatorname{dev}(\underline{\tau})\|=\frac{\sqrt{2}}{2}\left[\left(\tau_{1}-\tau_{2}\right)^{2}+\left(\tau_{2}-\tau_{3}\right)^{2}+\left(\tau_{1}-\tau_{3}\right)^{2}\right]^{\frac{1}{2}}$.

The isotropy assumption implies that the free energy $\psi$ can be expressed as a function of the principal elastic logarithmic stretches: $\psi\left(\boldsymbol{b}^{e}, \xi\right)=\widehat{\psi}\left(\underline{\varepsilon}^{e}, \xi\right)$ a symmetric function of the $\varepsilon_{A}^{e}$. With the preceding vector notation in hand, a straightforward manipulation of Eq. (15) gives the following stress-strain relations for the Kirchhoff principal stresses which are form-identical to those of infinitesimal strain case:

$$
\underline{\tau}=\frac{\partial \widehat{\psi}}{\partial \underline{\varepsilon}^{e}}\left(\underline{\varepsilon}^{e}, \xi\right)
$$

\subsection{General return mapping algorithm}

In this section, the essential ingredients of Simo's algorithm for multiplicative plasticity will be presented. The presentation proposed hereafter follows [38], see also [40] and [39].

\section{Trial elastic state}

Analogously to the infinitesimal case, the first -trial- step in the return mapping algorithm is the "elastic predictor". It corresponds physically to a state obtained by "freezing" the evolution of plastic flow and assuming the increment to be purely elastic. Consequently, the intermediate configuration remains unchanged, i.e.,

$$
C_{n+1}^{p^{t r}}=C_{n}^{p}
$$


Thus, with relationship (7) in hand, the trial elastic left Cauchy-Green strain is given by:

$$
\boldsymbol{b}_{t}^{e^{t r}}=\boldsymbol{F}_{t} \cdot \boldsymbol{C}_{n}^{p^{-1}} \cdot \boldsymbol{F}_{t}^{T}
$$

where the subscript $t$ refers to quantities evaluated within the time interval $\left.] t_{n}, t_{n+1}\right]$. Using the vector notations, the trial elastic state can be expressed in the principal axes $\boldsymbol{n}_{t}^{\operatorname{tr}(A)}$ (associated to $\boldsymbol{b}_{t}^{e^{t r}}$ ) as:

$$
\underline{\boldsymbol{\tau}}_{t}^{t r}=\frac{\partial \widehat{\psi}\left(\underline{\boldsymbol{\varepsilon}}_{t}^{\mathrm{tr}}, \xi_{t}^{t r}\right)}{\partial \underline{\boldsymbol{\varepsilon}}^{e}} ; q_{t}^{t r}=\frac{\partial \widehat{\psi}\left(\underline{\boldsymbol{\varepsilon}}_{t}^{e^{t r}}, \xi_{t}^{t r}\right)}{\partial \xi}
$$

where $\xi_{t}^{t r}=\xi_{n}$.

Return mapping algorithm in principal stresses

Simo [38] showed that using an exponential time integration algorithm of the flow rule enables to obtain the spectral decomposition of $\boldsymbol{b}_{t}^{e^{t r}}$ as:

$$
\boldsymbol{b}_{t}^{e^{t r}}=\sum_{A=1}^{3}\left(\lambda_{A}^{e}\right)^{2} \exp \left[2 \Delta \gamma_{t} \frac{\partial \widehat{f}\left(\tau_{1}, \tau_{2}, \tau_{3}, q\right)}{\partial \tau_{A}}\right] n_{t}^{(A)} \otimes n_{t}^{(A)}
$$

The principal directions $\boldsymbol{n}_{t}^{(A)}$ associated with the final state coincide with the principal directions $\boldsymbol{n}_{t}^{\operatorname{tr}(A)}$ defined by the trial elastic state. The algorithmic flow rule expressed in the principal axes $\boldsymbol{n}_{t}^{\operatorname{tr}(A)}$ is rephrased under the following form which is identical to the return mapping algorithm for models of infinitesimal plasticity:

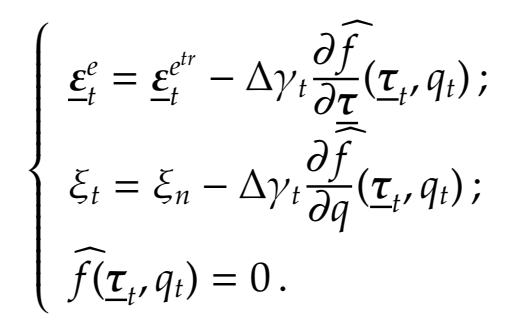

\subsection{Application to J2-flow theory}

The previous section summarized Simo's algorithm formulated in the principal stresses and strains with a general free energy function $\widehat{\psi}\left(\underline{\varepsilon}^{e}, \xi\right)$. In this section, with the preceding developments in hand, we consider the formulation of $J 2$-flow 
theory with isotropic hardening. It will be shown that in this particular context, the associated integration algorithm is exactly a straightforward extension of the radial return algorithm of infinitesimal J2-flow theory. We assume the following uncoupled form of the free energy function, quadratic in the principal elastic logarithmic stretches:

$$
\widehat{\psi}\left(\underline{\varepsilon}^{e}, \xi\right)=\frac{1}{2}\left(k-\frac{2}{3} G\right)\left(\varepsilon_{1}^{e}+\varepsilon_{2}^{e}+\varepsilon_{3}^{e}\right)^{2}+G\left[\left(\varepsilon_{1}^{e}\right)^{2}+\left(\varepsilon_{2}^{e}\right)^{2}+\left(\varepsilon_{3}^{e}\right)^{2}\right]+h(\xi) .
$$

where $k$ and $G$ are respectively the elastic bulk and shear moduli, and $h(\xi)$ is a function which characterizes the isotropic hardening in the material.

Then, the stress-strain relations in the principal axes are obtained by Eq. (18):

$$
\underline{\boldsymbol{\tau}}=\underline{\boldsymbol{a}}^{e l} \underline{\boldsymbol{\varepsilon}}^{e} ; \underline{\boldsymbol{e}}^{e l}=k \underline{\mathbf{1}} \underline{\mathbf{1}}^{T}+2 G\left[\underline{\boldsymbol{I}}-\frac{1}{3} \underline{\mathbf{1}} \underline{\mathbf{1}}^{T}\right]
$$

We consider the classical von Mises yield criterion formulated in terms of the principal Kirchhoff stresses as:

$$
\widehat{f}(\underline{\tau}, q)=\|\operatorname{dev}(\underline{\tau})\|-\sigma_{Y}-q(\xi) \leq 0,
$$

where $\sigma_{Y}$ is the flow stress and $q(\xi)$ is the hardening stress. The unit normal to the von Mises cylinder in the principal stress space is given by:

$$
\underline{N}=\frac{\partial \widehat{f}}{\partial \underline{\tau}}(\underline{\tau}, \xi)=\frac{3}{2} \frac{\operatorname{dev}(\underline{\tau})}{\|\operatorname{dev}(\underline{\tau})\|} .
$$

Premultiplying by $\underline{\boldsymbol{a}}^{\text {el }}$ the algorithmic flow rule (23), and using (25) along with (27) 
leads to the following form of the return mapping algorithm in the stress space:

$$
\left\{\begin{array}{l}
\underline{\boldsymbol{\tau}}_{n+1}^{t r}=\underline{\boldsymbol{a}}^{e l} \underline{\boldsymbol{e}}_{n+1}^{t r} \\
\underline{\boldsymbol{\tau}}_{n+1}=\underline{\boldsymbol{\tau}}_{n+1}^{t r}-2 G \Delta \gamma \underline{\boldsymbol{N}}\left(\underline{\boldsymbol{\tau}}_{n+1}\right) ; \\
\xi_{n+1}=\xi_{n}+\Delta \gamma ; \\
\underline{\boldsymbol{\varepsilon}}_{n+1}^{e}=\underline{\varepsilon}_{n+1}^{e^{t r}}-\Delta \gamma \underline{\boldsymbol{N}}\left(\underline{\boldsymbol{\tau}}_{n+1}\right) ; \\
\Delta \gamma{\widehat{f_{n+1}}}^{\prime r} 0, \Delta \gamma \geq 0, \widehat{f}_{n+1} \leq 0 .
\end{array}\right.
$$

Analogously to the infinitesimal theory, it is shown that the normal to the yield surface associated with the final state coincides with the normal defined by the trial state, i.e: $\underline{N}\left(\underline{\tau}_{n+1}\right)=\underline{N}\left(\underline{\tau}_{n+1}^{t r}\right)$, and the plastic correction step boils down to resolving the following familiar equation with the single scalar unknown $\Delta \xi$ :

$$
3 G \Delta \xi+q\left(\xi_{n}+\Delta \xi\right)+\sigma_{Y}-\left\|\operatorname{dev}\left(\underline{\tau}^{t r}\right)\right\|=0 .
$$

In order to write the incremental-secant formulation similarly to the infinitesimal strain increment format developed in [24], the set of Eqs. (27-28) is reformulated in the tensorial form. Indeed, since the principal directions $\boldsymbol{n}_{t}^{(A)}$ associated with the final state coincide with the principal directions $\boldsymbol{n}_{t}^{\operatorname{tr}(A)}$ associated with the trial state, the Kirchhoff stress tensor $\tau$ decomposed in Eq. (14), the final and trial logarithmic strain tensors

$$
\boldsymbol{\varepsilon}^{e}=\sum_{A=1}^{3} \varepsilon_{A}^{e} \boldsymbol{n}_{t}^{(A)} \otimes \boldsymbol{n}_{t}^{(A)}, \quad \boldsymbol{\varepsilon}^{e^{t r}}=\sum_{A=1}^{3} \varepsilon_{A}^{e^{t r}} \boldsymbol{n}_{t}^{(A)} \otimes \boldsymbol{n}_{t}^{(A)}
$$

and the normal to the von Mises surface (26)

$$
\boldsymbol{N}=\frac{3}{2} \frac{\operatorname{dev}(\tau)}{J_{2}(\tau)}=\sum_{A=1}^{3} v_{A} \boldsymbol{n}_{t}^{(A)} \otimes \boldsymbol{n}_{t}^{(A)}
$$

where $J_{2}(\tau)=\sqrt{\frac{3}{2} \operatorname{dev}(\tau): \operatorname{dev}(\tau)}$, are all expressed in the same principal directions. 
Therefore, the set of Eqs. (28) also reads

$$
\left\{\begin{array}{l}
\tau_{n+1}^{t r}=C^{e l}: \varepsilon_{n+1}^{e^{t r}} \\
\tau_{n+1}=\tau_{n+1}^{t r}-2 G \Delta \gamma N\left(\tau_{n+1}\right) \\
\xi_{n+1}=\xi_{n}+\Delta \gamma \\
\varepsilon_{n+1}^{e}=\varepsilon_{n+1}^{e^{t r}}-\Delta \gamma N\left(\tau_{n+1}\right) \\
\Delta \gamma f_{n+1}=0, \Delta \gamma \geq 0, f_{n+1} \leq 0
\end{array}\right.
$$

where

$$
\boldsymbol{C}^{e l}=k \boldsymbol{I} \otimes \boldsymbol{I}+2 G\left[\boldsymbol{I}-\frac{1}{3} \boldsymbol{I} \otimes \boldsymbol{I}\right]=3 k \boldsymbol{I}^{v o l}+2 G \boldsymbol{I}^{d e v},
$$

is the tensorial form of Eq. (25), with $I_{i j}=\delta_{i j}$ and $\mathcal{I}_{i j k l}=\frac{1}{2}\left(\delta_{i k} \delta_{j l}+\delta_{i l} \delta_{j k}\right)$.

\section{Mean-field homogenization (MFH) with the incremental-secant formulation}

Our approach for the homogenization of a nonlinear composite material at finite strains involves two key ingredients:

(i) First, we make use of the previous developments regarding the local hyperelastic-plastic constituents in order to bring the present finite deformation problem into a model which is form-similar to the infinitesimal theory.

(ii) Second, we transform the homogenization problem of the nonlinear composite material into that of a Linear Comparison Composite (LCC) and subsequently make use of classical results for linear composites. Our approach in this second step is based on a direct linearization of the constitutive behavior. The adopted linearization approach in the present work relies on the incremental-secant method following the work of [24], which enables to deal with a wide range of loading paths (e.g.: non monotonic and nonproportional loading conditions) while avoiding an isotropization step. The main ideas and steps of the incremental-secant formulation are recalled hereafter following the presentation of [24] with adaptation to the context of the finite strain regime. 


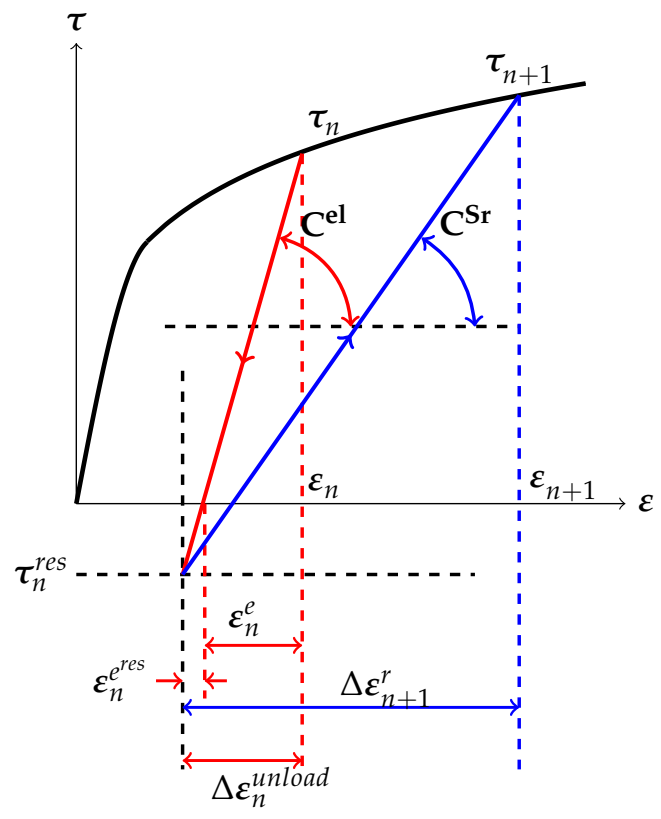

Figure 3: Schematics of the incremental-secant formulation in a local phase (subscript $\alpha$ is dropped for clarity) adapted from [24]. The residual-incremental-secant operator linearly relates the strain increment to the stress increment from an unloaded state.

\subsection{Linearization procedure with the incremental-secant approach}

We are concerned in this section with the local stress-strain relations in each phase $\omega_{\alpha}$ of the composite material. The incremental-secant formulation recently developed in [24] determines for each phase and at each increment a secant operator which links the strain increment to the stress increment from a virtually unloaded state, see Fig. 3. In the present proposal, and based on the local discrete constitutive Eq. (32), we use the logarithmic strain $\varepsilon$ and the Kirchhoff stress $\tau$ in order to transform the incremental-secant approach from the infinitesimal theory to the finite strain framework. Detailed needed pre-processing and post processing will be presented and discussed in a subsequent section.

\subsubsection{Kinematics}

Considering a time interval $\left[t_{n}, t_{n+1}\right]$, we suppose that the total strain tensor $\varepsilon_{n_{\alpha}}$, the Kirchhoff stress tensor $\tau_{n_{\alpha}}$ and all needed variables in each phase $\omega_{\alpha}$ to be known at the beginning of the time interval. In particular, in a first statistical moment MFH formulation, the per-phase volume averages of strain and stress 


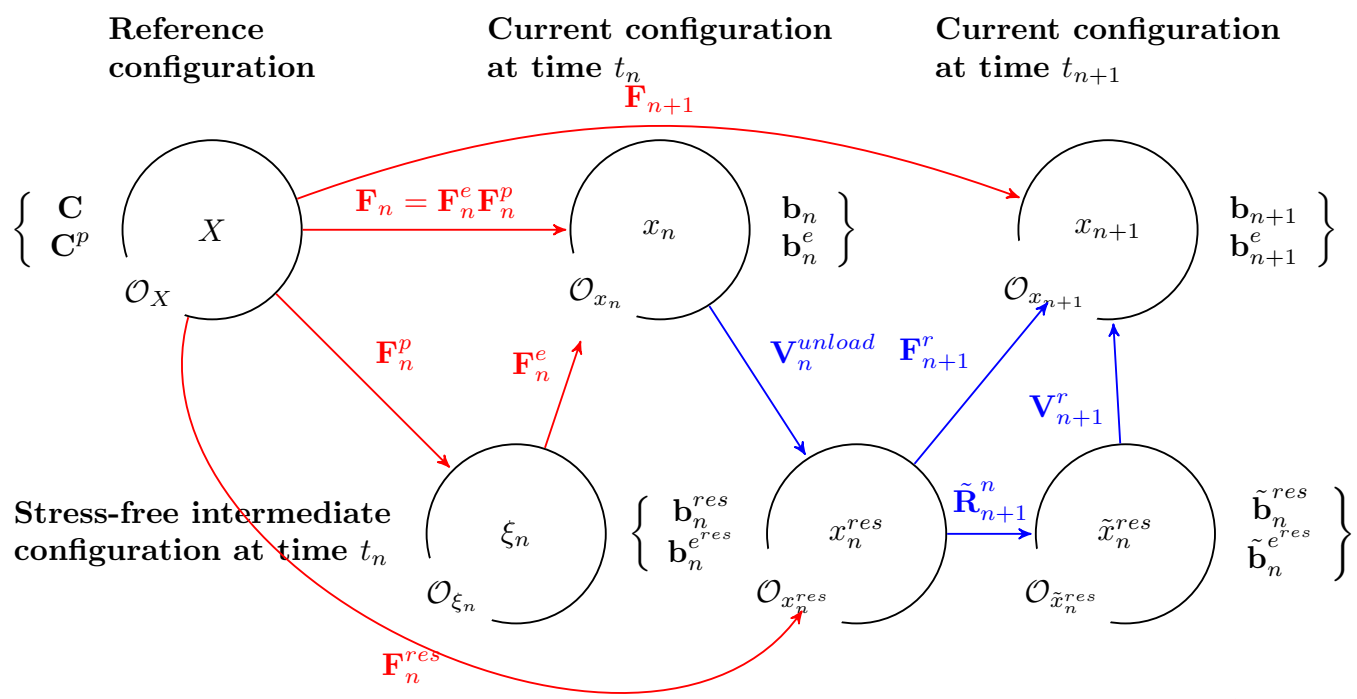

Virtually unloaded configurations

Figure 4: The trial elastic deformation gradient $\boldsymbol{F}_{n+1_{\alpha}}^{e^{t r}}=\boldsymbol{F}_{n+1_{\alpha}} \cdot \boldsymbol{F}_{n_{\alpha}}^{p^{-1}}$, is interpreted as a virtual elastic unloading $\boldsymbol{V}_{n_{\alpha}}^{\text {unload }}$ in the principal directions at configuration $t_{n}$ followed by an incremental deformation gradient $\boldsymbol{F}_{n+1_{\alpha}}^{r}=\boldsymbol{V}_{n+1_{\alpha}}^{r} \cdot \tilde{\boldsymbol{R}}_{n+1_{\alpha}}^{n}$. Strain tensors associated with the reference, current and virtually unloaded configurations are schematically indicated. Note that we used the subscript $n+1$ for the deformation gradients between configurations $t_{n}$ and $t_{n+1}$, and the subscript $n$ for the virtual unloading and the virtual residual configurations.

fields are supposed to be related by the point-wise constitutive model in the phase. Therefore, the return mapping algorithm summarized in Section 3.4 is applied on the average strain field in the phase $\omega_{\alpha}$. Thus, by construction, the average stress and elastic strain fields and Kirchhoff stress tensor share the same principal directions

$$
\boldsymbol{\tau}_{n_{\alpha}}=\sum_{A=1}^{3} \tau_{A_{n_{\alpha}}} \boldsymbol{n}_{n_{\alpha}}^{(A)} \otimes \boldsymbol{n}_{n_{\alpha}}^{(A)}, \quad \boldsymbol{b}_{n_{\alpha}}^{e}=\sum_{A=1}^{3}\left(\lambda_{A_{n_{\alpha}}}^{e}\right)^{2} \boldsymbol{n}_{n_{\alpha}}^{(A)} \otimes \boldsymbol{n}_{n_{\alpha}}^{(A)}
$$

Knowing the deformation tensor $\boldsymbol{F}_{n+1_{\alpha}}$, we are seeking the solution at time $t_{n+1}$, which reads

$$
\boldsymbol{\tau}_{n+1_{\alpha}}=\sum_{A=1}^{3} \tau_{A_{n+1 \alpha}} \boldsymbol{n}_{n+1_{\alpha}}^{(A)} \otimes \boldsymbol{n}_{n+1_{\alpha}}^{(A)}, \boldsymbol{b}_{n+1_{\alpha}}^{e}=\sum_{A=1}^{3}\left(\lambda_{A_{n+1 \alpha}}^{e}\right)^{2} \boldsymbol{n}_{n+1_{\alpha}}^{(A)} \otimes \boldsymbol{n}_{n+1_{\alpha}}^{(A)}
$$

Toward this end, the incremental-secant formulation decomposes the trial elastic deformation gradient $\boldsymbol{F}_{n+1_{\alpha}}^{e^{t r}}=\boldsymbol{F}_{n+1_{\alpha}} \cdot \boldsymbol{F}_{n_{\alpha}}^{p^{-1}}$ into an elastic unloading followed by 
an incremental deformation gradient, see Fig. 4. In particular, the elastic unloading is chosen so that it does not imply a rotation and can be written as the stretch tensor $\boldsymbol{V}_{n_{\alpha}}^{\text {unload }}$. It is decomposed in the principal directions at configuration $t_{n}$ as $\boldsymbol{V}_{n_{\alpha}}^{\text {unload }}=\sum_{A=1}^{3} \lambda_{A_{n_{\alpha}}}^{\text {unload }} \boldsymbol{n}_{n_{\alpha}}^{(A)} \otimes \boldsymbol{n}_{n_{\alpha}}^{(A)}$. The incremental deformation gradient is thus decomposed into a stretch tensor and a rotation tensor, with $\boldsymbol{F}_{n+1_{\alpha}}^{r}=\boldsymbol{V}_{n+1_{\alpha}}^{r} \cdot \tilde{\boldsymbol{R}}_{n+1_{\alpha}}^{n}$, so that the elastic predictor is finally expressed as

$$
\boldsymbol{F}_{n+1_{\alpha}}^{e^{t r}}=\boldsymbol{V}_{n+1_{\alpha}}^{r} \cdot \tilde{\boldsymbol{R}}_{n+1_{\alpha}}^{n} \cdot \boldsymbol{V}_{n_{\alpha}}^{\text {unload }} \cdot \boldsymbol{F}_{n_{\alpha}}^{e}
$$

in which $\boldsymbol{V}_{n+1_{\alpha}}^{r}=\sum_{A=1}^{3} \lambda_{A_{n+1_{\alpha}}}^{r} \boldsymbol{n}_{n+1_{\alpha}}^{(A)} \otimes \boldsymbol{n}_{n+1_{\alpha}}^{(A)}$ is defined as the trial incremental stretch from the unloaded configuration, and in which the tensor

$$
\tilde{\boldsymbol{R}}_{n+1_{\alpha}}^{n}=\sum_{A=1}^{3} \boldsymbol{n}_{n+1_{\alpha}}^{(A)} \otimes \boldsymbol{n}_{n_{\alpha}}^{(A)}
$$

brings the principal directions at configuration $t_{n}$ to the principal directions at configuration $t_{n+1}$. As a result, the principal directions associated to the virtual Kirchhoff stress in configuration $\tilde{\boldsymbol{x}}_{n}^{\text {res }}$ are those of the Kirchhoff stress in configuration $\boldsymbol{x}_{n+1}$. The amplitude of the unloading in the phase $\omega_{\alpha}$ will be defined during the homogenization process in order to unload elastically the composite material.

\subsubsection{Virtual unloading}

Since it does not involve a plastic flow, applying the virtual elastic unloading

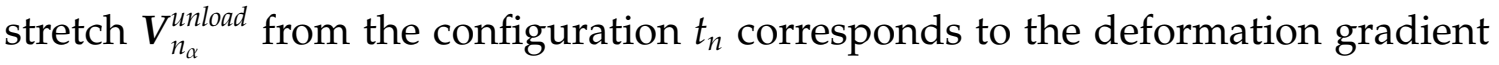
$\boldsymbol{F}_{n_{\alpha}}^{\text {res }}=\boldsymbol{V}_{n_{\alpha}}^{\text {unload }} \cdot \boldsymbol{F}_{n_{\alpha}}=\boldsymbol{V}_{n_{\alpha}}^{\text {unload }} \cdot \boldsymbol{F}_{n_{\alpha}}^{e} \cdot \boldsymbol{F}_{n_{\alpha}}^{p}$. As a result, the elastic left Cauchy-Green tensor reads

$$
\boldsymbol{b}_{n_{\alpha}}^{e^{\text {res }}}=\boldsymbol{V}_{n_{\alpha}}^{\text {unload }} \cdot \boldsymbol{b}_{n_{\alpha}}^{e} \cdot \boldsymbol{V}_{n_{\alpha}}^{\text {unload }}=\sum_{A=1}^{3}\left(\lambda_{A_{n_{\alpha}}}^{e}\right)^{2}\left(\lambda_{A_{n_{\alpha}}}^{\text {unload }}\right)^{2} \boldsymbol{n}_{n_{\alpha}}^{(A)} \otimes \boldsymbol{n}_{n_{\alpha}}^{(A)}
$$

and the residual elastic logarithmic strain reads

$$
\boldsymbol{\varepsilon}_{n_{\alpha}}^{e^{r e s}}=\sum_{A=1}^{3}\left[\ln \left(\lambda_{A_{n_{\alpha}}}^{\text {unload }}\right)+\ln \left(\lambda_{A_{n_{\alpha}}}^{e}\right)\right] \boldsymbol{n}_{n_{\alpha}}^{(A)} \otimes \boldsymbol{n}_{n_{\alpha}}^{(A)}=\Delta \boldsymbol{\varepsilon}_{n_{\alpha}}^{\text {unload }}+\boldsymbol{\varepsilon}_{n_{\alpha}}^{e}
$$


where

$$
\Delta \boldsymbol{\varepsilon}_{n_{\alpha}}^{\text {unload }}=\sum_{A=1}^{3} \ln \left(\lambda_{A_{n_{\alpha}}}^{\text {unload }}\right) \boldsymbol{n}_{n_{\alpha}}^{(A)} \otimes \boldsymbol{n}_{n_{\alpha}}^{(A)} .
$$

This unloading is illustrated in the one dimensional case in Fig. 3.

Applying the system of Eqs. (28) or (32) on this last result yields $\underline{\boldsymbol{\tau}}_{n_{\alpha}}^{\text {res }}=\underline{\boldsymbol{a}}^{e l} \underline{\underline{\varepsilon}}_{n_{\alpha}}^{\text {res }}=$ $\underline{\boldsymbol{\tau}}_{n_{\alpha}}+\underline{\boldsymbol{a}}^{\text {el }} \Delta \underline{\varepsilon}_{n_{\alpha}}^{\text {unload }}$. Therefore, using the definition (34), the residual Kirchhoff stress tensor is obtained using the elastic tensor (33) following

$$
\boldsymbol{\tau}_{n_{\alpha}}^{r e s}=\sum_{A=1}^{3} \tau_{A_{n_{\alpha}}}^{r e s} \boldsymbol{n}_{n_{\alpha}}^{(A)} \otimes \boldsymbol{n}_{n_{\alpha}}^{(A)}=\boldsymbol{\tau}_{n_{\alpha}}+\boldsymbol{C}^{e l}: \Delta \boldsymbol{\varepsilon}_{n_{\alpha}}^{\text {unlooad }}
$$

\subsubsection{Residual state}

After having applied the unloading part of the trial elastic deformation gradient $\boldsymbol{F}_{n+1_{\alpha}}^{t^{t r}}(36)$, the incremental deformation gradient $\boldsymbol{F}_{n+1_{\alpha}}^{r}=\boldsymbol{V}_{n+1_{\alpha}}^{r} \cdot \tilde{\boldsymbol{R}}_{n+1_{\alpha}}^{n}$ is applied in two steps. First the rotation $\tilde{\boldsymbol{R}}_{n+1_{\alpha}}^{n}$ in order to define a residual state in the principal directions at configuration $t_{n+1}$, and then the trial stretch increment $V_{n+1_{\alpha}}^{r}$.

Considering the initial rotation $\tilde{\boldsymbol{R}}_{n+1_{\alpha}}^{n}$ applied at the unloaded configuration, since the transformation does not involve plasticity, the corresponding deformation gradient reads $\tilde{\boldsymbol{F}}_{n_{\alpha}}^{r e s}=\tilde{\boldsymbol{R}}_{n+1_{\alpha}}^{n} \cdot \boldsymbol{F}_{n_{\alpha}}^{\text {res }}=\tilde{\boldsymbol{R}}_{n+1_{\alpha}}^{n} \cdot \boldsymbol{V}_{n_{\alpha}}^{\text {unload }} \cdot \boldsymbol{F}_{n_{\alpha}}^{e} \cdot \boldsymbol{F}_{n_{\alpha}}^{p}$. As a result, the elastic left Cauchy-Green tensor reads

$$
\tilde{\boldsymbol{b}}_{n_{\alpha}}^{e^{r e s}}=\tilde{\boldsymbol{F}}_{n_{\alpha}}^{r e s} \cdot \boldsymbol{C}_{n_{\alpha}}^{p^{-1}} \cdot \tilde{\boldsymbol{F}}_{n_{\alpha}}^{r e s^{T}}=\tilde{\boldsymbol{R}}_{n+1_{\alpha}}^{n} \cdot \boldsymbol{b}_{n_{\alpha}}^{\boldsymbol{e}^{r e s}} \cdot \tilde{\boldsymbol{R}}_{n+1_{\alpha}}^{n^{T}}=\sum_{A=1}^{3}\left(\lambda_{A_{n_{\alpha}}}^{e^{r e s}}\right)^{2} \boldsymbol{n}_{n+1_{\alpha}}^{(A)} \otimes \boldsymbol{n}_{n+1_{\alpha}}^{(A)},
$$

with $\lambda_{A_{n_{\alpha}}}^{e^{\text {res }}}=\lambda_{A_{n_{\alpha}}}^{e} \lambda_{A_{n_{\alpha}}}^{\text {unload. }}$.

The corresponding rotated elastic logarithmic strain $\tilde{\varepsilon}_{n_{\alpha}}^{\text {res }}$ is obtained according to

$$
\tilde{\boldsymbol{\varepsilon}}_{n_{\alpha}}^{r^{r e s}}=\sum_{A=1}^{3} \ln \left(\lambda_{A_{n_{\alpha}}}^{e^{r e s}}\right) \boldsymbol{n}_{n+1_{\alpha}}^{(A)} \otimes \boldsymbol{n}_{n+1_{\alpha}}^{(A)}=\tilde{\boldsymbol{R}}_{n+1_{\alpha}}^{n} \boldsymbol{\varepsilon}_{n_{\alpha}}^{\boldsymbol{e}^{r e s}} \tilde{\boldsymbol{R}}_{n+1}^{n^{T}}
$$

Since $\tilde{\boldsymbol{b}}_{n_{\alpha}}^{\tilde{e}^{\text {res }}}$ and $\boldsymbol{b}_{n_{\alpha}}^{\boldsymbol{e}^{\text {res }}}$ share the same eigenvalues, the principal stress components $\underline{\boldsymbol{\tau}}_{n_{\alpha}}^{\text {res }}$ 
are the same and the system of Eqs. (28) or (32) simplifies into

$$
\tilde{\boldsymbol{\tau}}_{n_{\alpha}}^{r e s}=\sum_{A=1}^{3} \tau_{A_{n_{\alpha}}}^{\boldsymbol{r e s}_{n+1_{\alpha}}} \boldsymbol{n}_{n+1_{\alpha}}^{(A)} \otimes \boldsymbol{n}_{n+1_{\alpha}}^{(A)} \cdot \tilde{\boldsymbol{\tau}}_{n_{\alpha}}^{r e s} \cdot \tilde{\boldsymbol{R}}_{n+1_{\alpha}}^{n^{T}} .
$$

\subsubsection{The predictor-corrector and return mapping schemes}

The Kirchhoff stress tensor at configuration $t_{n+1}$ is obtained by introducing the residual state in the predictor-corrector algorithm (28) or (32), as illustrated in Fig. 5. When considering the incremental-secant approach similar to Eq. (36), we have, see Fig. 4,

$$
\boldsymbol{F}_{n+1_{\alpha}}=\boldsymbol{V}_{n+1_{\alpha}}^{r} \cdot \tilde{\boldsymbol{R}}_{n+1_{\alpha}}^{n} \cdot \boldsymbol{V}_{n_{\alpha}}^{\text {unload }} \cdot \boldsymbol{F}_{n_{\alpha}}=\boldsymbol{V}_{n+1_{\alpha}}^{r} \cdot \tilde{\boldsymbol{R}}_{n+1_{\alpha}}^{n} \cdot \boldsymbol{V}_{n_{\alpha}}^{\text {unload }} \cdot \boldsymbol{F}_{n_{\alpha}}^{e} \cdot \boldsymbol{F}_{n_{\alpha}}^{p},
$$

or again using Eq. (42)

$$
\boldsymbol{b}_{n+1_{\alpha}}^{e^{t r}}=\boldsymbol{F}_{n+1_{\alpha}} C_{n_{\alpha}}^{p^{-1}} \boldsymbol{F}_{n+1_{\alpha}}^{T}=\boldsymbol{V}_{n+1_{\alpha}}^{r} \cdot \tilde{\boldsymbol{b}}_{n_{\alpha}}^{\tilde{r e s}^{r e s}} \cdot \boldsymbol{V}_{n+1_{\alpha}}^{r}
$$

Since $\tilde{\boldsymbol{b}}_{n_{\alpha}}^{e^{r e s}}$ and $\boldsymbol{V}_{n+1_{\alpha}}^{r}$ have the same principal directions, the trial stretch tensor is expressed as

$$
\boldsymbol{\varepsilon}_{n+1_{\alpha}}^{e^{r r}}=\sum_{A=1}^{3}\left[\ln \left(\lambda_{A_{n+1_{\alpha}}}^{r}\right)+\ln \left(\lambda_{A_{n_{\alpha}}}^{e^{r \varepsilon s}}\right)\right] \boldsymbol{n}_{n+1_{\alpha}}^{(A)} \otimes \boldsymbol{n}_{n+1_{\alpha}}^{(A)}=\Delta \boldsymbol{\varepsilon}_{n+1_{\alpha}}^{r}+\tilde{\boldsymbol{\varepsilon}}_{n_{\alpha}}^{r \epsilon s}
$$

where

$$
\Delta \boldsymbol{\varepsilon}_{n+1_{\alpha}}^{r}=\sum_{A=1}^{3} \ln \left(\lambda_{A_{n+1_{\alpha}}}^{r}\right) \boldsymbol{n}_{n+1_{\alpha}}^{(A)} \otimes \boldsymbol{n}_{n+1_{\alpha}}^{(A)}
$$

is illustrated in the one dimensional case in Fig. 3.

Applying an elastic loading according to the system of Eqs. (28) or (32) yields the trial principal stress components $\underline{\boldsymbol{\tau}}_{n+1_{\alpha}}^{t r}=\underline{\boldsymbol{a}}^{e l} \underline{\varepsilon}_{n+1_{\alpha}}^{\text {etr }}=\underline{\boldsymbol{\tau}}_{n_{\alpha}}^{\text {res }}+\underline{\boldsymbol{a}}^{e l} \Delta \underline{\boldsymbol{\varepsilon}}_{n+1_{\alpha}}^{r}$, or under the tensorial form

$$
\boldsymbol{\tau}_{n+1_{\alpha}}^{t r}=\boldsymbol{C}_{\alpha}^{e l}: \varepsilon_{n+1_{\alpha}}^{t r}=\tilde{\boldsymbol{\tau}}_{n_{\alpha}}^{r e s}+\Delta \boldsymbol{\tau}_{n+1_{\alpha}}^{t r}
$$




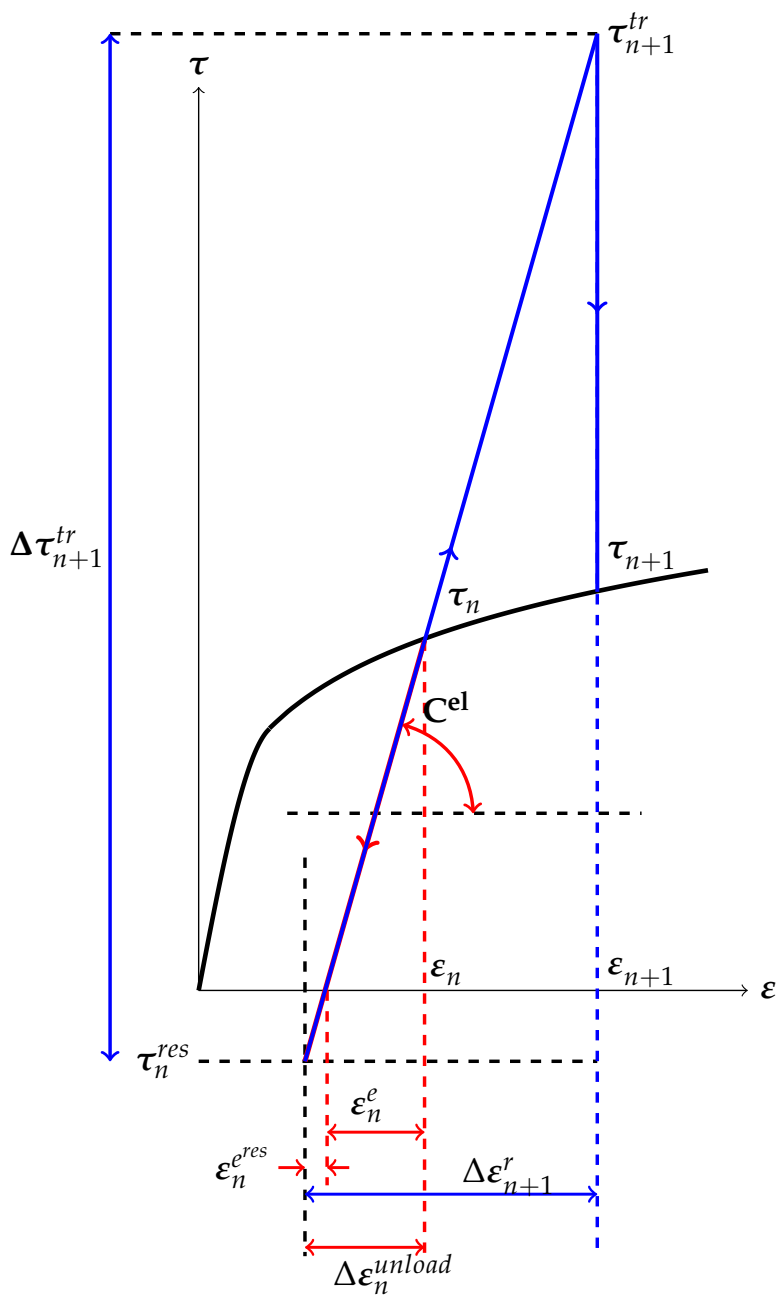

(a)

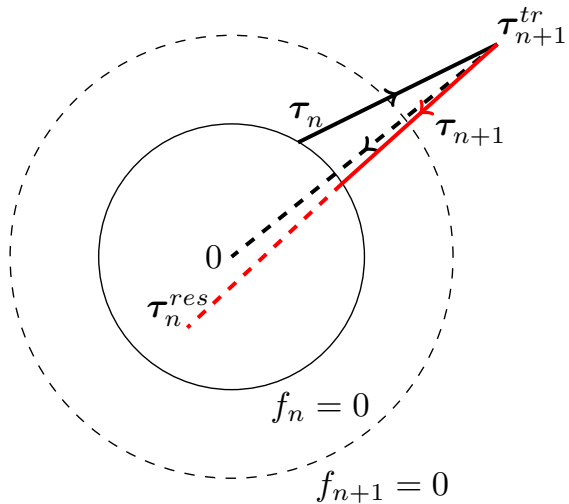

(b)

Figure 5: The predictor-corrector scheme: (a) the trial stress is computed from an elastic increment (i.e.: $\left.\boldsymbol{C}^{S r}=\boldsymbol{C}^{e l}\right)$, (b) the plastic correction brings the stress back to the yield surface $\left(f_{n+1}=0\right)$ : both the classical radial return mapping and the modified direction of the plastic flow adopted in this model are presented. 
where $\Delta \tau_{n+1_{\alpha}}^{t r}=C_{\alpha}^{e l}: \Delta \varepsilon_{n+1_{\alpha}}^{r}$. If the trial stress satisfies the yield condition:

$$
f_{n+1_{\alpha}}^{t r i a l}\left(\tau_{n+1_{\alpha}}^{t r}, q_{n_{\alpha}}\right) \leq 0
$$

then the actual increment is fully elastic and the trial state is indeed the solution, i.e. $\tau_{n+1_{\alpha}}=\tau_{n+1_{\alpha}}^{t r}$.

In the case of plastic flow, using Simo [38]'s exponential time integration algorithm of the flow rule (22) yields the tensorial form

$$
\boldsymbol{b}_{n+1_{\alpha}}^{e}=\exp \left(-2 \Delta \gamma N\left(\tau_{n+1_{\alpha}}\right)\right) \boldsymbol{b}_{n+1_{\alpha}}^{t^{t r}} .
$$

Therefore, using Eq. (47) allows the system of Eqs. (28) or (32) to be rewritten as

$$
\varepsilon_{n+1_{\alpha}}^{e}=\varepsilon_{n+1_{\alpha}}^{e t r}-\Delta \gamma N\left(\tau_{n+1_{\alpha}}\right)=\Delta \varepsilon_{n+1_{\alpha}}^{r}+\tilde{\boldsymbol{\varepsilon}}_{n_{\alpha}}^{e^{r e s}}-\Delta \gamma \boldsymbol{N}\left(\tau_{n+1_{\alpha}}\right) .
$$

The principal stress components follow from $\underline{\boldsymbol{\tau}}_{n+1_{\alpha}}=\underline{\boldsymbol{\tau}}_{n+1_{\alpha}}^{t r}-2 G \Delta \gamma \underline{\boldsymbol{N}}\left(\underline{\boldsymbol{\tau}}_{n+1_{\alpha}}\right)=$ $\underline{\tau}_{n_{\alpha}}^{r e s}+\underline{\boldsymbol{a}}^{e l} \Delta \underline{\varepsilon}_{n_{\alpha}}^{r}-2 G \Delta \gamma \underline{\boldsymbol{N}}\left(\underline{\tau}_{n+1_{\alpha}}\right)$, or under the tensorial form

$$
\boldsymbol{\tau}_{n+1_{\alpha}}=\tilde{\boldsymbol{\tau}}_{n_{\alpha}}^{r e s}+\Delta \boldsymbol{\tau}_{n+1_{\alpha}}^{r} \text { with } \Delta \boldsymbol{\tau}_{n+1_{\alpha}}^{r}=\Delta \boldsymbol{\tau}_{n+1_{\alpha}}^{t r}-2 G \Delta \gamma \mathbf{N}\left(\boldsymbol{\tau}_{n+1_{\alpha}}\right)
$$

Following [24], we choose a modified plastic flow direction given by:

$$
N_{n+1_{\alpha}}=\frac{3}{2} \frac{\operatorname{dev}\left(\tau_{n+1_{\alpha}}-\tilde{\tau}_{n_{\alpha}}^{r e s}\right)}{J_{2}\left(\tau_{n+1_{\alpha}}-\tilde{\tau}_{n_{\alpha}}^{r e s}\right)}=\frac{3}{2} \frac{\operatorname{dev}\left(\boldsymbol{C}^{S r}: \Delta \boldsymbol{\varepsilon}_{n+1_{\alpha}}^{r}\right)}{J_{2}\left(C^{S r}: \Delta \boldsymbol{\varepsilon}_{n+1_{\alpha}}^{r}\right)},
$$

which satisfies $N: N=\frac{3}{2}$. This implies that the plastic correction is directed along $\Delta \tau_{\alpha}^{r}$ and not along $\tau_{\alpha}$ as in the classical Eq. (27). As discussed in [24], for a single phase material this corresponds to a first-order approximation in terms of $\Delta \varepsilon_{n+1_{\alpha}}$, ensuring the accuracy of the scheme for small enough increments. Finally, when $\operatorname{dev}\left(\tilde{\tau}_{n_{\alpha}}^{r e s}\right)=0$, the classical case is retrieved. The approximation used in the calculation of $N$ is schematically depicted in Fig. 5-(b). It will be shown later that this approximation allows to directly obtain an isotropic incremental-secant 
operator. The trial flow direction is also introduced as

$$
N_{n+1_{\alpha}}^{t r}=\frac{3}{2} \frac{\operatorname{dev}\left(\tau_{n+1_{\alpha}}^{t r}-\tilde{\tau}_{n_{\alpha}}^{r e s}\right)}{J_{2}\left(\tau_{n+1_{\alpha}}^{t r}-\tilde{\tau}_{n_{\alpha}}^{r e s}\right)}=\frac{3}{2} \frac{\operatorname{dev}\left(C^{e l}: \Delta \varepsilon_{n+1_{\alpha}}^{r}\right)}{J_{2}\left(C^{e l}: \Delta \varepsilon_{n+1_{\alpha}}^{r}\right)},
$$

and it was shown in [24] that $N_{n+1_{\alpha}}^{t r}=N_{n+1_{\alpha}}$. Using this last result, Eqs. (54) and (55) lead to the following result

$$
J_{2}\left(\tau_{n+1_{\alpha}}-\tilde{\tau}_{n_{\alpha}}^{r e s}\right)+3 G \Delta \gamma=J_{2}\left(\tau_{n+1_{\alpha}}^{t r}-\tilde{\tau}_{n_{\alpha}}^{r e s}\right)
$$

The plastic correction consists in solving the last equation together with $f\left(\tau_{n+1_{\alpha}}, q_{n+1_{\alpha}}\right)=$ 0 .

\subsubsection{Incremental-secant-operator}

With a view of the definition of the future LCC, Eq. (53) is rewritten under the form

$$
\Delta \tau_{n+1_{\alpha}}^{r}=C_{\alpha}^{S r}: \Delta \varepsilon_{n+1_{\alpha}}^{r},
$$

where $\mathcal{C}_{\alpha}^{S r}$ is the residual-incremental-secant operator of the linear comparison material.

On the one hand, if there is no plastic flow between the configuration $t_{n}$ and $t_{n+1}$, then $\boldsymbol{C}_{\alpha}^{S r}=\mathcal{C}_{\alpha}^{e l}$. On the other hand, in case of plastic flow, substituting Eq. (53) into Eq. (57) and using Eq. (55) yield the following explicit expression of the incremental-secant operator:

$$
\boldsymbol{C}_{\alpha}^{S r}=\boldsymbol{C}_{\alpha}^{e l}-3 G \Delta \gamma \frac{\mathcal{I}^{d e v}: \boldsymbol{C}_{\alpha}^{e l}}{J_{2}\left(C_{\alpha}^{e l}: \Delta \varepsilon_{n+1_{\alpha}}^{r}\right)}
$$

Since $\boldsymbol{C}_{\alpha}^{e l}$ is isotropic, the residual-incremental-secant operator $\boldsymbol{C}_{\alpha}^{S r}$ of the linear comparison material is also isotropic and can be recast as:

$$
\boldsymbol{C}_{\alpha}^{S r}=3 k^{S r} \boldsymbol{I}^{v o l}+2 G^{S r} \boldsymbol{I}^{d e v},
$$


with

$$
k_{\alpha}^{S r}=k_{\alpha}^{e l} \text {, and } G_{\alpha}^{S r}=\frac{J_{2}\left(\Delta \tau_{n+1_{\alpha}}^{r}\right)}{3 \sqrt{\frac{2}{3} \operatorname{dev}\left(\Delta \varepsilon_{n+1_{\alpha}}^{r}\right): \operatorname{dev}\left(\Delta \varepsilon_{n+1_{\alpha}}^{r}\right)}} .
$$

In the particular case of an elastic loading in the phase $\alpha$, it is seen from Eq. (60 that the incremental-secant operator reduces to the elastic tensor.

When embedding this incremental-secant phase linearization during an homogenization process, as described in the next section, it has been shown in [24] that in the context of small deformations, for hard inclusions embedded in elastoplastic matrix, the residual stress in the matrix phase (but neither the residual strain nor the residual values in the inclusions phase) had to be canceled before applying the secant homogenization, unless second statistical moments were accounted for as in [26]. However, in the presented large deformation approach, the residual stress tensor $\tilde{\tau}_{n_{M}}^{r e s}$ in the matrix phase does not appear in the radial return mapping algorithm. Thefore, this modification is introduced when defining the incremental-secant operator used by the LCC, which becomes

$$
\tau_{n+1_{\alpha}}=\mathcal{C}_{\alpha}^{S 0}: \Delta \varepsilon_{n+1_{\alpha}}^{r}
$$

where $\boldsymbol{C}_{\alpha}^{S 0}$ is the zero-incremental-secant operator of the linear comparison material. In that case, during a plastic flow, the definition of the normal direction is the usual one, i.e.

$$
N_{n+1_{\alpha}}^{t r}=\frac{3}{2} \frac{\operatorname{dev}\left(\tau_{n+1_{\alpha}}^{t r}\right)}{J_{2}\left(\tau_{n+1_{\alpha}}^{t r}\right)}=N_{n+1_{\alpha}},
$$

and the isotropic zero-incremental-secant operator reads

$$
\boldsymbol{C}_{\alpha}^{S 0}=3 k^{S 0} \mathcal{I}^{v o l}+2 G^{S 0} \mathcal{I}^{d e v},
$$

with

$$
k_{\alpha}^{S 0}=\frac{\operatorname{tr}\left(\tau_{n+1_{\alpha}}^{r}\right)}{3 \operatorname{tr}\left(\Delta \varepsilon_{n+1_{\alpha}}^{r}\right)} \text {, and } G_{\alpha}^{S 0}=\frac{J_{2}\left(\tau_{n+1_{\alpha}}^{r}\right)}{3 \sqrt{\frac{2}{3} \operatorname{dev}\left(\Delta \varepsilon_{n+1_{\alpha}}^{r}\right): \operatorname{dev}\left(\Delta \varepsilon_{n+1_{\alpha}}^{r}\right)}} \text {. }
$$




\subsection{Homogenization procedure with the incremental-secant approach}

In this section, we consider a two-phase composite made of (at least one) hyperelastic-plastic phases $\omega_{\alpha}$. We first present the assumptions required to extend the incremental-secant MFH scheme to finite strains. We then detail first how to evaluate the unloaded state of the composite material and then how to perform the MFH.

\subsubsection{Definition of the Linear Comparison Composite}

The so-called Linear Comparison Composite is defined through the residual- or zero-incremental-secant operators, which are chosen by design uniform per phase $\alpha$ and will be denoted $\overline{\boldsymbol{C}}_{\alpha}^{S}$, holding for either $\overline{\boldsymbol{C}}_{\alpha}^{S r}$ or $\overline{\boldsymbol{C}}_{\alpha}^{S 0}$, as developed in Section 4.1. It is also assumed that this fictitious composite has a spatial distribution of the phases and geometries that coincides with that of the actual non-linear composite.

The incremental-secant MFH is characterized by the following equations, in which a modeling assumption consists in applying the averaging rules of micromechanics on the logarithmic strains on the current configuration and not on the deformation gradients on the reference configuration:

- A phase averaging of the Cauchy stress tensor

$$
\overline{\boldsymbol{\sigma}}_{n+1}=f_{n+1_{M}} \boldsymbol{\sigma}_{n+1_{M}}+f_{n+1_{I}} \boldsymbol{\sigma}_{n+1_{I}}
$$

- A phase averaging of the incremental logarithmic strain from a virtually unloaded state

$$
\Delta \bar{\varepsilon}_{n+1}^{r}=f_{n+1_{M}} \Delta \varepsilon_{n+1_{M}}^{r}+f_{n+1_{I}} \Delta \varepsilon_{n+1_{I}}^{r}
$$

- The definition of the strain concentration tensor $\boldsymbol{B}^{\varepsilon}$ such that

$$
\Delta \varepsilon_{n+1_{I}}^{r}=B^{\varepsilon}\left(I, C_{M}^{S}, C_{I}^{S}\right): \Delta \varepsilon_{n+1_{M}}^{r} ;
$$

The expression of $\boldsymbol{B}^{\varepsilon}$ corresponds in this work to the one for Mori-Tanaka 
model and reads:

$$
\boldsymbol{B}^{\varepsilon}=\left\{\mathcal{I}+\mathcal{S}:\left(\mathcal{C}_{M}^{S}\right)^{-1}:\left[\mathcal{C}_{I}^{S}-\mathcal{C}_{M}^{S r}\right]\right\}^{-1}
$$

where $\mathcal{S}\left(I, \boldsymbol{C}_{M}^{S}\right)$ designates Eshelby's tensor, which depends on the shape and orientation of inclusion $(I)$, and the incremental-secant stiffness of the matrix comparison material. The evaluations of the Eshelby tensor and of the strain localization tensor (68) rely on the use of the incremental-secant operators $\boldsymbol{C}_{\alpha}^{S}$ (59) as LCC operators in each phase $\omega_{\alpha}$, which happen to be naturally isotropic. This is one of the main advantages of the incremental-secant method, which is preserved in the finite strain formalism: the isotropization step of the LCC operator required by both the affine and incremental-tangent methods [23] is avoided.

In these equations, the subscripts $M$ and $I$ designate the matrix and inclusions phases respectively, stresses and strains with subscript $\alpha$ designate corresponding volume averages over the current configuration of phase $\omega_{\alpha}$, and $f_{n+1_{\alpha}}$ is the volume fraction of phase $\alpha$ at $t_{n+1}$. Within this formulation, the evolution of the microstructure during the deformation process is accounted for, on the one hand through the evolution of the volume fractions $f_{n+1_{\alpha}}$, and on the other hand, through the evolution of the shape and orientation of inclusion $(I)$ when evaluating the Eshelby tensor. Details will be given in the algorithmic description in Section 4.2.3. If linear displacements corresponding to a macro logarithmic strain are applied to the RVEs boundary, then to the authors knowledge, there is no proof that this applied strain is equal to the volume average of the micro logarithmic strains on neither the current nor the reference configurations of the RVE [41]. Applying the averaging rules of micro-mechanics on the logarithmic strains remains an assumption, but, despite its apparent roughness, it will be shown that the obtained results, at least at the macroscopic scale, are very satisfying.

In Eq. (66), it is also assumed that the LCC is subjected to a logarithmic strain increment $\Delta \bar{\varepsilon}_{n+1}^{r}$ which is deduced from the unloaded residual state and which is 
different from the actual strain increment applied to the actual RVE. Considering a time interval $\left[t_{n}, t_{n+1}\right]$, we suppose that the macroscopic strain gradient tensors $\bar{F}$ are known in the configurations $t_{n}$ and $t_{n+1}$. The macroscopic left Cauchy Green strain tensor in the two configurations follow

$$
\overline{\boldsymbol{b}}_{n}=\overline{\boldsymbol{F}}_{n} \cdot \overline{\boldsymbol{F}}_{n}^{T}, \quad \overline{\boldsymbol{b}}_{n+1}=\overline{\boldsymbol{F}}_{n+1} \cdot \overline{\boldsymbol{F}}_{n+1}^{T},
$$

and have a spectral decomposition $\overline{\boldsymbol{b}}_{n+1}$ with eigenvalues $\left(\lambda_{A}\right)^{2}$ and principal directions $\boldsymbol{n}^{(A)}$ as

$$
\overline{\boldsymbol{b}}_{n}=\sum_{A=1}^{3}\left(\lambda_{A_{n}}\right)^{2} \boldsymbol{n}_{n}^{(A)} \otimes \boldsymbol{n}_{n}^{(A)}, \quad \overline{\boldsymbol{b}}_{n+1}=\sum_{A=1}^{3}\left(\lambda_{A_{n+1}}\right)^{2} \boldsymbol{n}_{n+1}^{(A)} \otimes \boldsymbol{n}_{n+1}^{(A)}
$$

At configuration $t_{n}$ we also know the Kirchhoff stress tensor $\overline{\boldsymbol{\tau}}_{n}$ and we are seeking the solution $\bar{\tau}_{n+1}$ at time $t_{n+1}$. In order to apply the incremental-secant formulation presented in Section 4.1 in the phases $\omega_{\alpha}$, we decompose the macroscale deformation gradient $\overline{\boldsymbol{F}}_{n+1}$ in a similar way as performed for the phases in Section 4.1, i.e.

$$
\overline{\boldsymbol{F}}_{n+1}=\overline{\boldsymbol{F}}_{n+1}^{r} \cdot \overline{\boldsymbol{V}}_{n}^{\text {unload }} \cdot \overline{\boldsymbol{F}}_{n},
$$

in which $\overline{\boldsymbol{V}}_{n}^{\text {unload }}=\sum_{A=1}^{3} \lambda_{A_{n}}^{\text {unload }} \boldsymbol{n}_{n}^{(A)} \otimes \boldsymbol{n}_{n}^{(A)}$ is defined as an unloading stretch, and in which the incremental deformation gradient is decomposed following

$$
\overline{\boldsymbol{F}}_{n+1}^{r}=\overline{\boldsymbol{V}}_{n+1}^{r} \cdot \tilde{\boldsymbol{R}}_{n+1}^{n} \cdot
$$

In this last equation $\overline{\boldsymbol{V}}_{n+1}^{r}=\sum_{A=1}^{3} \lambda_{A_{n+1}}^{r} \boldsymbol{n}_{n+1}^{(A)} \otimes \boldsymbol{n}_{n}^{(A)}$ is defined as the incremental stretch from the virtually unloaded configuration and $\tilde{\boldsymbol{R}}_{n+1}^{n}$ is defined as

$$
\tilde{\boldsymbol{R}}_{n+1}^{n}=\sum_{A=1}^{3} \boldsymbol{n}_{n+1}^{(A)} \otimes \boldsymbol{n}_{n}^{(A)}
$$

and brings the principal directions of the composite material at configuration $t_{n}$ to the principal directions at configuration $t_{n+1}$. 


\subsubsection{Virtual unloading}

The virtual elastic unloading of the composite material consists in applying the stretch $\overline{\boldsymbol{V}}_{n}^{\text {unload }}$ from the configuration $t_{n}$, corresponding to the deformation gradient $\overline{\boldsymbol{F}}_{n}^{\text {res }}=\overline{\boldsymbol{V}}_{n}^{\text {unload }} \cdot \overline{\boldsymbol{F}}_{n}$. As a result, the left Cauchy-Green tensor reads

$$
\overline{\boldsymbol{b}}_{n}^{\text {res }}=\overline{\boldsymbol{V}}_{n}^{\text {unload }} \cdot \overline{\boldsymbol{b}}_{n} \cdot \overline{\boldsymbol{V}}_{n}^{\text {unload }}=\sum_{A=1}^{3}\left(\lambda_{A_{n}}\right)^{2}\left(\lambda_{A_{n}}^{\text {unload }}\right)^{2} \boldsymbol{n}_{n}^{(A)} \otimes \boldsymbol{n}_{n}^{(A)}
$$

and the residual elastic logarithmic strain reads

$$
\overline{\boldsymbol{\varepsilon}}_{n}^{\text {res }}=\sum_{A=1}^{3} \ln \left(\lambda_{A_{n}}^{r e s}\right) \boldsymbol{n}_{n}^{(A)} \otimes \boldsymbol{n}_{n}^{(A)}=\sum_{A=1}^{3}\left[\ln \left(\lambda_{A_{n}}^{\text {unload }}\right)+\ln \left(\lambda_{A_{n}}\right)\right] \boldsymbol{n}_{n}^{(A)} \otimes \boldsymbol{n}_{n}^{(A)}=\Delta \overline{\boldsymbol{\varepsilon}}_{n}^{\text {unload }}+\overline{\boldsymbol{\varepsilon}}_{n}
$$

where $\lambda_{A_{n}}^{\text {res }}=\lambda_{A_{n}}^{\text {unload }} \lambda_{A_{n}}$ and where $\Delta \overline{\boldsymbol{\varepsilon}}_{n}^{\text {unload }}=\sum_{A=1}^{3} \ln \left(\lambda_{A_{n}}^{\text {unload }}\right) \boldsymbol{n}_{n}^{(A)} \otimes \boldsymbol{n}_{n}^{(A)}$.

The virtual unloading is obtained by assuming a purely elastic unloading process of the composite material from the configuration at time $t_{n}$, yielding

$$
\bar{\varepsilon}_{n}^{\text {res }}=\bar{\varepsilon}_{n}-\Delta \bar{\varepsilon}_{n}^{\text {unload }}=\bar{\varepsilon}_{n}-\left(\overline{\boldsymbol{C}}^{e l}\right)^{-1}: \overline{\boldsymbol{\tau}}_{n}
$$

In this last equation, we made the assumption that the principal directions of the Kirchhoff stress tensor $\bar{\tau}_{n}$ are close to the ones of the strain tensor $\bar{\varepsilon}_{n}$. Indeed, contrarily to the case of the phases in which the radial return mapping algorithm leads to such relations in terms of the elastic strains, this is not necessarily true after performing homogenization at the composite material level. However, this assumption allows performing the elastic unloading without an iterative process. Equation (66) is rewritten to reach a zero-stress at the composite level, leading to

$$
\overline{\boldsymbol{\tau}}_{n}^{r e s}=0=\overline{\boldsymbol{\sigma}}_{n}^{r e s}=f_{n_{M}} \boldsymbol{\sigma}_{n_{M}}^{r e s}+f_{n_{I}} \boldsymbol{\sigma}_{n_{I}}^{r e s}=f_{n_{M}} \frac{\boldsymbol{\tau}_{n_{M}}^{r e s}}{J_{n_{M}}^{r e s}}+f_{n_{I}} \frac{\boldsymbol{\tau}_{n_{I}}^{r e s}}{J_{n_{I}}^{r e s}}
$$

with

$$
\tau_{n_{\alpha}}^{r e s}=\tau_{n_{\alpha}}-C_{\alpha}^{e l}: \Delta \varepsilon_{n_{\alpha}}^{\text {unload }}(\alpha=M ; I)
$$


In these equations, $J_{n_{\alpha}}$ is the determinant of the average deformation gradient over the phase $\omega_{\alpha}$. Equation (65) is rewritten during the unloading

$$
\Delta \bar{\varepsilon}_{n}^{\text {unload }}=f_{n_{M}} \Delta \varepsilon_{n_{M}}^{\text {unload }}+f_{n_{I}} \Delta \varepsilon_{n_{I}}^{\text {unload }} .
$$

Finally, Eq. (67) gives a relation between the unloading mean strain increments per phase:

$$
\Delta \varepsilon_{n_{I}}^{\text {unload }}=B^{\varepsilon}\left(I, C_{M}^{e l}, C_{I}^{e l}\right): \Delta \varepsilon_{n_{M}}^{\text {unload }}
$$

This enables to compute the elastic residual strains in the inclusions and matrix phases from:

$$
\begin{aligned}
& \varepsilon_{n_{I}}^{\text {res }}=\varepsilon_{n_{I}}^{e}-\Delta \varepsilon_{n_{I}}^{\text {unload }}=\varepsilon_{n_{I}}^{e}-B^{\varepsilon}:\left[f_{n_{I}} \boldsymbol{B}^{\varepsilon}+f_{n_{M}} \mathcal{I}\right]^{-1}: \Delta \bar{\varepsilon}_{n}^{\text {unload }} ; \\
& \varepsilon_{n_{M}}^{\text {res }}=\varepsilon_{n_{M}}^{e}-\Delta \varepsilon_{n_{M}}^{\text {unload }}=\varepsilon_{n_{M}}^{e}-\left[f_{n_{I}} \boldsymbol{B}^{\varepsilon}+f_{n_{M}} \mathcal{I}\right]^{-1}: \Delta \bar{\varepsilon}_{n}^{\text {unload }} .
\end{aligned}
$$

From the decomposition (71), using the expression (70) and the rotation (73), since the different stretch tensors are expressed in the same principal directions, the macroscopic left Cauchy Green strain tensor (69) reads

$$
\overline{\boldsymbol{b}}_{n+1}=\overline{\boldsymbol{V}}_{n+1}^{r} \cdot \tilde{\boldsymbol{R}}_{n+1}^{n} \cdot \overline{\boldsymbol{V}}_{n}^{\text {unload }} \cdot \overline{\boldsymbol{F}}_{n} \cdot \overline{\boldsymbol{F}}_{n}^{T} \cdot \overline{\boldsymbol{V}}_{n}^{\text {unload }} \cdot \tilde{\boldsymbol{R}}_{n+1}^{n^{T}} \cdot \overline{\boldsymbol{V}}_{n+1}^{r}=\sum_{A=1}^{3}\left(\lambda_{A_{n+1}}^{r}\right)^{2}\left(\lambda_{A_{n}}^{\text {res }}\right)^{2} \boldsymbol{n}_{n+1}^{(A)} \otimes \boldsymbol{n}_{n+1}^{(A)},
$$

and the logarithmic strain tensor reads

$$
\overline{\boldsymbol{\varepsilon}}_{n+1}=\sum_{A=1}^{3}\left[\ln \left(\lambda_{A_{n+1}}^{r}\right)+\ln \left(\lambda_{A_{n}}^{r e s}\right)\right] \boldsymbol{n}_{n+1}^{(A)} \otimes \boldsymbol{n}_{n+1}^{(A)}=\Delta \overline{\boldsymbol{\varepsilon}}_{n+1}^{r}+\tilde{\boldsymbol{\varepsilon}}_{n}^{r e s},
$$

where

$$
\Delta \bar{\varepsilon}_{n+1}^{r}=\sum_{A=1}^{3} \ln \left(\lambda_{A_{n+1}}^{r}\right) n_{n+1}^{(A)} \otimes n_{n+1}^{(A)}
$$

is the incremental macroscopic strain tensor, and

$$
\tilde{\boldsymbol{\varepsilon}}_{n}^{r e s}=\sum_{A=1}^{3} \ln \left(\lambda_{A_{n}}^{r e s}\right) \boldsymbol{n}_{n+1}^{(A)} \otimes \boldsymbol{n}_{n+1}^{(A)}=\tilde{\boldsymbol{R}}_{n+1}^{n} \bar{\varepsilon}_{n}^{r e s} \tilde{\boldsymbol{R}}_{n+1}^{n^{T}}
$$


is residual strain expressed in the current principal directions.

\subsubsection{Incremental-secant homogenization}

This macroscopic strain increment is computed from Eqs. (83) and (76) as

$$
\Delta \bar{\varepsilon}_{n+1}^{r}=\bar{\varepsilon}_{n+1}-\bar{\varepsilon}_{n}^{r e s},
$$

which allows the system of Eqs. (65-67) to be solved.

This homogenization procedure, which allows updating the macroscopic stress, is described in the following.

- Known data: $\overline{\boldsymbol{F}}_{n}, \overline{\boldsymbol{F}}_{n+1}$, all history variables at configuration $t_{n}$ are also known for both the composite material and for each phase $\omega_{\alpha}$, including the residual elastic strain tensors $\varepsilon_{n_{\alpha}}^{r e s}(81)$ and corresponding residual stress tensors $\boldsymbol{\tau}_{n_{\alpha}}^{\text {res }}$ of the phases $\omega_{\alpha}$, expressed in the principal directions $\boldsymbol{n}_{n_{\alpha}}^{(A)}$, and the residual strain tensor $\bar{\varepsilon}_{n}^{\text {res }}(76)$ of the composite material, expressed in the principal directions $\boldsymbol{n}_{n}^{(A)}$.

- Compute the macroscopic left Cauchy Green strain tensor:

$$
\overline{\boldsymbol{b}}_{n+1}=\overline{\boldsymbol{F}}_{n+1} \cdot \overline{\boldsymbol{F}}_{n+1}^{T} \cdot
$$

- Perform the spectral decomposition of $\overline{\boldsymbol{b}}_{n+1}$, from which eigenvalues are denoted $\left(\lambda_{A_{n+1}}\right)^{2}$ and principal directions $\boldsymbol{n}_{n+1}^{(A)}$, which allows evaluating the logarithmic strain

$$
\overline{\boldsymbol{\varepsilon}}_{n+1}=\sum_{A=1}^{3} \ln \left(\lambda_{A_{n+1}}\right) \boldsymbol{n}_{n+1}^{(A)} \otimes \boldsymbol{n}_{n+1}^{(A)}
$$

- Evaluate the composite rotation tensor $\tilde{\boldsymbol{R}}_{n+1}^{n}$ from Eq. (73), and push the residual strain $\bar{\varepsilon}_{n}^{r e s}$ in the current principal directions to obtain $\tilde{\varepsilon}_{n}^{r e s}$ using Eq. (85).

- Compute the macroscopic strain increment $\Delta \bar{\varepsilon}_{n+1}^{r}$ to be applied to the LCC from Eq. (83). 
- Initialization : the strain increment in the inclusions phase is initialized from the macroscopic one:

$$
\Delta \varepsilon_{n+1_{I}}^{r} \leftarrow \Delta \bar{\varepsilon}_{n+1}^{r} .
$$

- Iteration (i): A Newton-Raphson iterative process is applied over the strain increment in the inclusions phase (upper indices (i) are omitted for clarity)

1) Compute the mean strain increment in the matrix phase:

$$
\Delta \varepsilon_{n+1_{M}}^{r}=\frac{\Delta \bar{\varepsilon}_{n+1}^{r}-f_{n_{I}} \Delta \varepsilon_{n+1_{I}}^{r}}{f_{n_{M}}} .
$$

2) Trial elastic state (for each phase $\alpha=I, M$ ):

i Compute the spectral decomposition (48) of $\Delta \varepsilon_{n+1_{\alpha}}^{r}$ to obtain the principal logarithmic strain $\ln \left(\lambda_{A_{n+1} \alpha}^{r}\right)$ and the principal directions $\boldsymbol{n}_{n+1_{\alpha}}^{(A)}$.

ii Evaluate the rotation increment $\tilde{\boldsymbol{R}}_{n+1_{\alpha}}^{n}$ in the phase using Eq. (37), and rotate the residual logarithmic elastic strain $\varepsilon_{n_{\alpha}}^{r e s}$ and stress $\tau_{n_{\alpha}}^{r e s}$ to obtain their respective counterparts $\tilde{\varepsilon}_{n_{\alpha}}^{\text {res }}$ and $\tilde{\tau}_{n_{\alpha}}^{\text {res }}$ in the principal directions $\boldsymbol{n}_{n+1_{\alpha}}^{(A)}$, using respectively Eqs. (43) and (44).

iii Compute the trial principal elastic logarithmic stretches $\varepsilon_{n+1_{\alpha}}^{t t r}$ in the phase $\alpha$ from Eq. (47).

iv Compute the Kirchhoff trial stress $\tau_{n+1_{\alpha}}^{t r}$ from Eq. (49).

3) Call the constitutive box of each phase $\alpha$ (described in Section 4.1.4) with $\Delta \varepsilon_{n+1_{\alpha}}^{r}$ and $\tau_{n+1_{\alpha}}^{t r}$ as entries. The output is the updated stress $\tau_{n+1_{\alpha}}$, the internal variables at $t_{n+1}$ and either the residual-incremental-secant operator $\boldsymbol{C}_{\alpha}^{S r}$ computed from Eq. (59) or the zero-incremental-secant operator $\boldsymbol{C}_{\alpha}^{S 0}$ computed from Eq. (63).

4) Update the deformation gradient matrix of phase $\omega_{\alpha}$ following

$$
\boldsymbol{F}_{n+1_{\alpha}}=\boldsymbol{V}_{n+1_{\alpha}}^{r} \tilde{\boldsymbol{R}}_{n+1_{\alpha}}^{n} \boldsymbol{V}_{n_{\alpha}}^{\text {unload }} \cdot \boldsymbol{F}_{n_{\alpha}}
$$


where $V_{n+1_{\alpha}}^{r}$ and $V_{n_{\alpha}}^{\text {unload }}$ are evaluated from respectively $\Delta \varepsilon_{n+1_{\alpha}}^{r}$ and $\Delta \varepsilon_{n_{\alpha}}^{\text {unload }}$.

5) Compute the Eshelby's tensor $\mathcal{S}_{n+1}\left(I_{n+1}, \boldsymbol{C}_{n+1_{M}}^{S}\right)$ in terms of the updated matrix material behavior $\mathcal{C}_{n+1_{M}}^{S}$ and of the new inclusion geometry designated by $I_{n+1}$. The geometry is updated using the inclusion deformation gradient $\boldsymbol{F}_{n+1_{I}}$ evolution as follows. Defining the initial ellipsoid matrix $\boldsymbol{A}_{0_{I}}=\boldsymbol{R}_{0_{I}} \cdot \operatorname{diag}\left(\frac{1}{a_{1_{0}}^{2}}, \frac{1}{a_{2_{0}}^{2}}, \frac{1}{a_{30}^{2}}\right) \cdot \boldsymbol{R}_{0_{I^{\prime}}}^{T}$, where $\boldsymbol{R}_{0_{I}}$ defines the original orientation matrix and $a_{i_{0}}$ the initial ellipsoid aspect ratios, implies $\boldsymbol{X}^{T} \cdot \boldsymbol{A}_{0_{I}} \cdot \boldsymbol{X}=1$ and the updated ellipsoid matrix $\boldsymbol{A}_{n+1_{I}}$, which satisfies $\boldsymbol{x}^{T} \cdot \boldsymbol{A}_{n+1_{I}} \cdot \boldsymbol{x}=1$, follows from $\boldsymbol{A}_{n+1_{I}}=\boldsymbol{F}_{n+1_{I}}^{-T} \cdot \boldsymbol{A}_{n+1_{I}} \cdot \boldsymbol{F}_{n+1_{I}}^{-1}$. The updated aspect ratios $a_{i_{n+1}}$ and orientation matrix $\boldsymbol{R}_{n+1_{I}}$ follow from the eigenvalues and eigenvectors of $\boldsymbol{A}_{n+1_{I}}$.

6) Check compatibility of the strain increment in the matrix $\Delta \varepsilon_{n+1_{M}}^{r}$. In order to solve these MFH iterations, Eq. (67) is rewritten as $r=0$ where $r$ is the stress residual in the inclusions phase, see Appendix $B$ for details. During the time step $\left[t_{n} ; t_{n+1}\right]$, the strain increment $\Delta \bar{\varepsilon}_{n+1}^{r}$ is constant and the stress residual can be evaluated as

$$
\boldsymbol{r}=\boldsymbol{C}_{n+1_{M}}^{\mathcal{S}}:\left[\Delta \varepsilon_{n+1_{I}}^{r}-\frac{1}{f_{n_{M}}} \mathcal{S}_{n+1}^{-1}:\left(\Delta \varepsilon_{n+1_{I}}^{r}-\Delta \bar{\varepsilon}_{n+1}^{r}\right)\right]-\mathcal{C}_{n+1_{I}}^{\mathcal{S}}: \Delta \boldsymbol{\varepsilon}_{n+1_{I}}^{r}
$$

- If $\|r\| \leq$ TOL, then exit the loop.

- Else: a new iteration has to be performed (go to step 1) and the strain increment in the inclusions phase is corrected following:

$$
\Delta \varepsilon_{n+1_{I}}^{r} \leftarrow \Delta \varepsilon_{n+1_{I}}^{r}-J^{-1}: r
$$

The Jacobian $J$ matrix is computed at constant $\Delta \bar{\varepsilon}_{n+1}^{r}$, such that $\delta \boldsymbol{r}=J: \delta \Delta \varepsilon_{I}^{r}$. The details about the Newton-Raphson procedure and the computation of the Jacobian matrix are given in Appendix $B$.

\section{After convergence:}


- Compute the principal elastic logarithmic stretches in the different phases $\varepsilon_{\alpha}^{e}$ at $t_{n+1}$ from Eq. (32-d).

- Compute the inverse of the elastic left Cauchy-Green strain in both phases $\alpha$ :

$$
\left(\boldsymbol{b}_{n+1_{\alpha}}^{e}\right)^{-1}=\sum_{A=1}^{3} \exp \left[-2 \varepsilon_{A_{n+1_{\alpha}}}^{e}\right] \boldsymbol{n}_{n+1_{\alpha}}^{(A)} \otimes \boldsymbol{n}_{n+1_{\alpha}}^{(A)}
$$

- Update each phase's volume fractions

$$
f_{n+1_{\alpha}}=\frac{J_{n+1_{\alpha}} f_{0_{\alpha}}}{J_{n+1_{M}} f_{0_{M}}+J_{n+1_{I}} f_{0_{I}}},
$$

with $J_{n+1_{\alpha}}^{r}=\operatorname{det} V_{n+1_{\alpha}}^{r}=\prod_{A} \lambda_{A_{n+1_{\alpha}}}^{r}$ and $J_{n+1_{\alpha}}=J_{n+1_{\alpha}}^{r} J_{n_{\alpha}}^{r e s}$.

- Compute the macroscopic stress update:

$$
\left\{\begin{array}{l}
\overline{\boldsymbol{\sigma}}_{n+1}=f_{n+1_{M}} \frac{\boldsymbol{\tau}_{n+1_{M}}}{J_{n+1_{M}}}+f_{n+1_{I}} \frac{\boldsymbol{\tau}_{n+1_{I}}}{J_{n+1_{I}}} ; \\
\overline{\boldsymbol{\tau}}_{n+1}=\bar{J}_{n+1} \overline{\boldsymbol{\sigma}}_{n+1} .
\end{array}\right.
$$

- Unloading step: Knowing the principal directions $\boldsymbol{n}_{n+1}^{(A)}$, the residual variables $\varepsilon_{n+1_{\alpha}}^{\text {res }}, \bar{r}_{n+1}^{r e s}, \tau_{n+1_{\alpha}}^{r e s}$ are obtained from the virtual elastic unloading applied at the composite material level, see Section 4.2.2, by solving the set of Eqs. (76)-(81).

\section{Assessment of the model}

The proposed incremental-secant MFH formulation was tested for several twophase particulate composites and porous materials. We compare its predictions to reference results obtained from Finite Element (FE) simulations. All the presented MFH results have converged with respect to the time step increment, the residualincremental-secant operator is considered in the inclusions phase and the zeroincremental-secant operator in the matrix phase. 


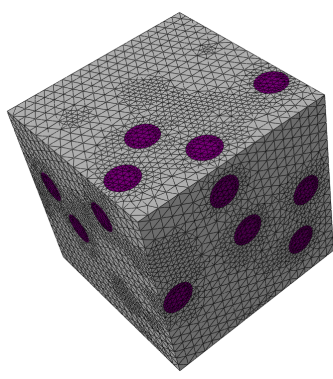

(a) $10 \%$ RVE

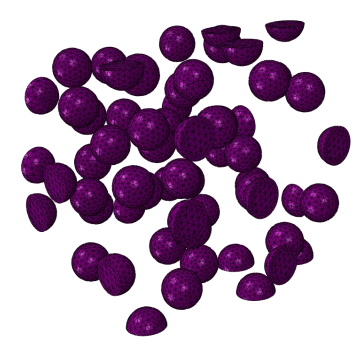

(b) $10 \%$ inclusions

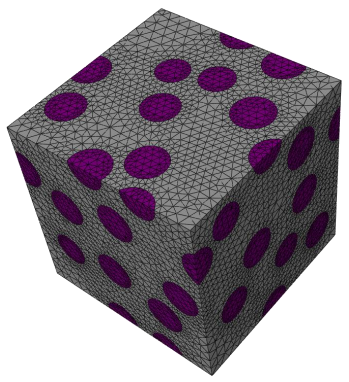

(c) $20 \%$ RVE

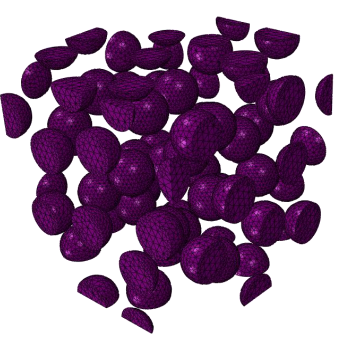

(d) $20 \%$ inclusions

Figure 6: RVEs for FE analysis of a matrix material reinforced with spherical inclusions: (a) and (b) 10\%-inclusion reinforced matrix; (c) and (d) 20\%-inclusion reinforced matrix.

\subsection{Metal matrix composites with spherical inclusions}

First we consider spherical inclusion-reinforced elasto-plastic matrix with successively two initial inclusions volume fractions of $f_{0_{1}}=0.1$ and $f_{0_{1}}=0.2$. The RVEs are illustrated in Fig. 6 and contain respectively 40 and 50 inclusions. Finite Element computations were conducted with the commercial solver Abaqus. The RVEs were meshed using 10-node tetrahedra (C3D10) and comprise about $1.92 \times 10^{5}$ and $3.02 \times 10^{5}$ elements for respectively $f_{0_{I}}=0.1$ and $f_{0_{I}}=0.2$.

The MFH incremental-secant procedure is assessed for a metal matrix composite (MMC), which is made of a $J_{2}$ elasto-plastic matrix reinforced by stiffer elastic inclusions. The matrix phase presents an isotropic hardening described by a power law followed by a bi-linear hardening 1 :

$$
q(\xi)= \begin{cases}h_{1} \xi^{n} & \text { if } \xi \leq \xi_{0} \\ \xi_{0}^{n}+h_{2}\left(\xi-\xi_{0}\right) & \text { if } \xi_{0}<\xi \leq 10 \xi_{0} \\ \xi_{0}^{n}+9 h_{2} \xi_{0} & \text { if } 10 \xi_{0}<\xi\end{cases}
$$

The material parameters for the matrix and the inclusions' phases are:

- Inclusions: $E=400 \mathrm{GPa}, v=0.2$;

- Matrix: $E=75 \mathrm{GPa}, v=0.3, \sigma_{Y}=75 \mathrm{MPa}, h_{1}=416 \mathrm{MPa}, n=0.3895, \xi_{0}=0.17$, and

\footnotetext{
${ }^{1}$ The linear hardening results from the extrapolation-like entry of an Abaqus file.
} 


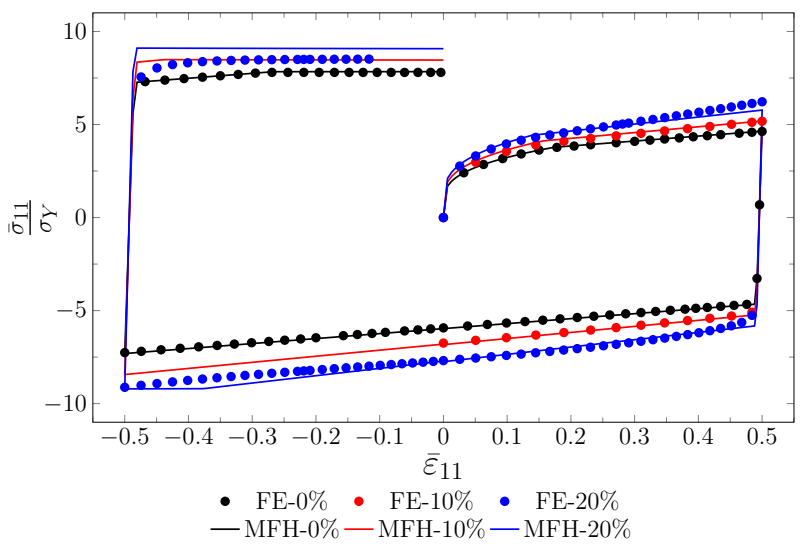

(a) Macro stresses

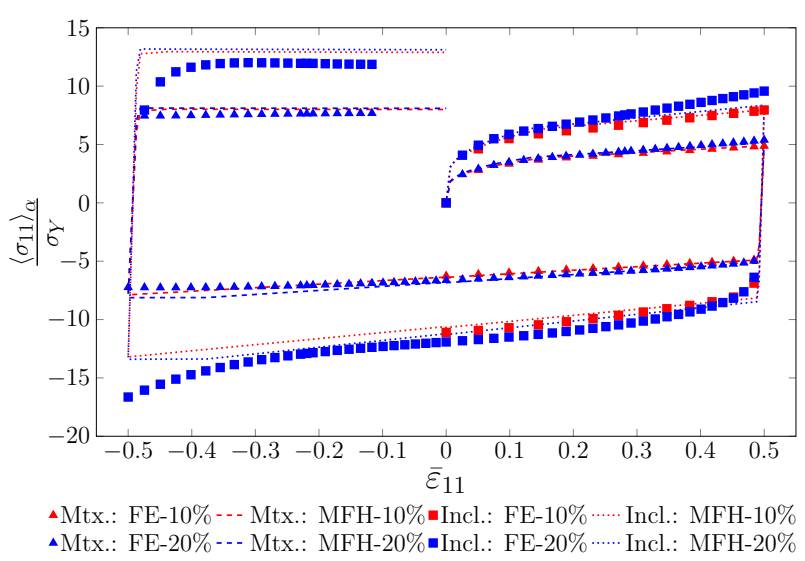

(b) Phase average stresses

Figure 7: Effective responses (a) and per-phase responses (b) of three MMCs ( $0 \%, 10 \%$ and 20\% of stiff elastic inclusions embedded in an elasto-plastic matrix) under macro uni-axial tension. Normalized homogenized stresses vs. macro strain. The response of the matrix is also provided for comparison.

$$
h_{2}=200 \mathrm{MPa} .
$$

These parameters correspond to an aluminum alloy reinforced with stiff ceramic particles.

Figure 7(a) shows the effective response under uni-axial tension-compression cyclic loading of the two considered metal matrix composites as well as the response of the matrix without reinforcement (i.e. $f_{0_{l}}=0$ ). The latter case is considered in order to verify that the large deformation constitutive elasto-plastic formulation considered in the MFH is consistent with the one used by Abaqus for the RVE simulations. Unless stated otherwise, all the curves present the evolution of the Cauchy stress in term of the logarithmic strain. Figure 7(a) shows that the predictions of the macroscopic response delivered by the MFH-incremental-secant formulation are in good agreement with the reference results from FE computations for the composite material containing $10 \%$ of inclusions. For the composite material containing $20 \%$ of inclusions, the composite material response shows a discrepancy at the higher strain stages, in particular during the compressiontension part of the cycle.

The phases' responses predicted by the Mean-Field (MFH) model and FE analysis are presented in Fig. 7(b). One could notice that the phase responses are 


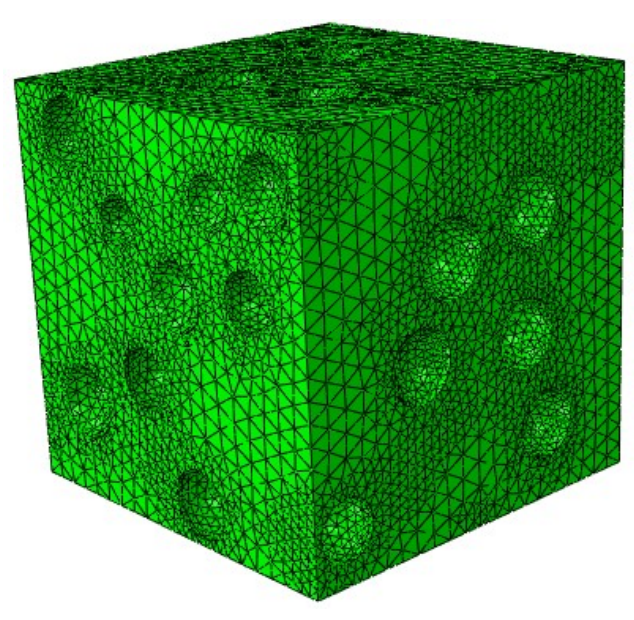

(a) $10 \%$ porosity

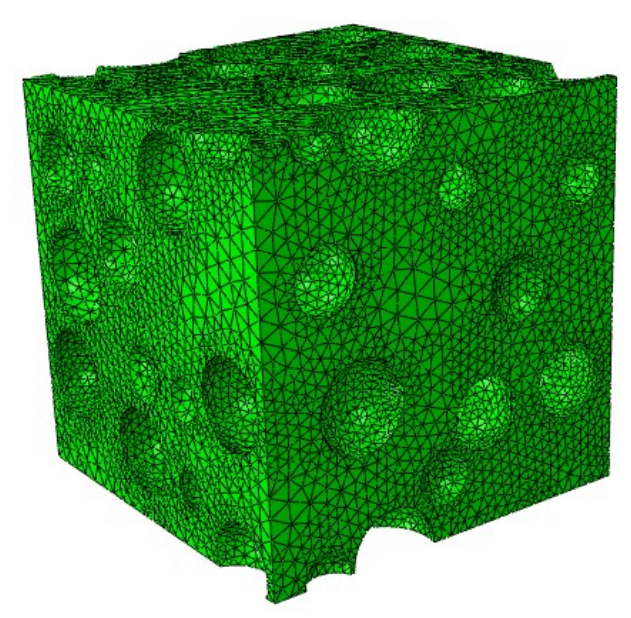

(b) $25 \%$ porosity

Figure 8: RVEs of a porous metallic matrix containing $10 \%$ and $25 \%$ of initially spherical cavities.

accurately captured by the mean-field model for the composite material containing $10 \%$ of inclusions. However, at the higher strain, inaccuracies are related to the prediction of the inclusion response for the composite material comprising $20 \%$ of inclusions. The stress in the inclusions phase is underestimated at the higher tensile, and in particular at the higher compressive stress, affecting the macro-stress response as seen in Fig. 7(a).

\subsection{Porous material}

A porous metallic matrix is now considered. The matrix has the same material properties as the aluminum alloy considered previously for the MMC. Instead of reinforcements, we consider two RVEs wih initially spherical voids dispersed in the matrix phase with $10 \%$ and $25 \%$ of initial porosity, as illustrated in Fig. 8 .

The FE simulations performed in this study are based on cubic RVEs consisting of a random dispersion of respectively 48 and 50 initially spherical cavities having different sizes embedded in a continuum matrix for the $10 \%$ and $25 \%$ of porous RVEs. The RVE micro-structure is periodic along the cube axes allowing to apply periodic boundary conditions (PBCs). The RVEs were meshed using modified 10-node tetrahedra hybrid elements with linear pressure (C3D10MH), with respectively $1.47 \times 10^{5}$ and $2.24 \times 10^{5}$ elements. 


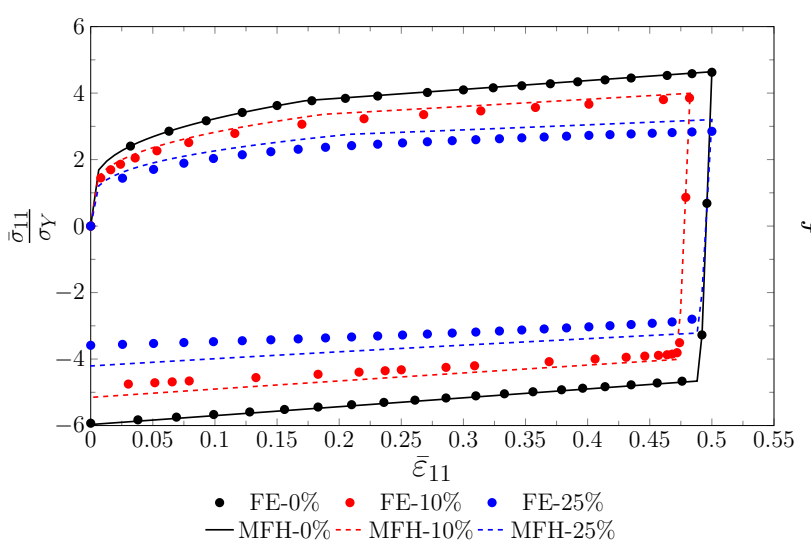

(a) Macro stresses; tensile test

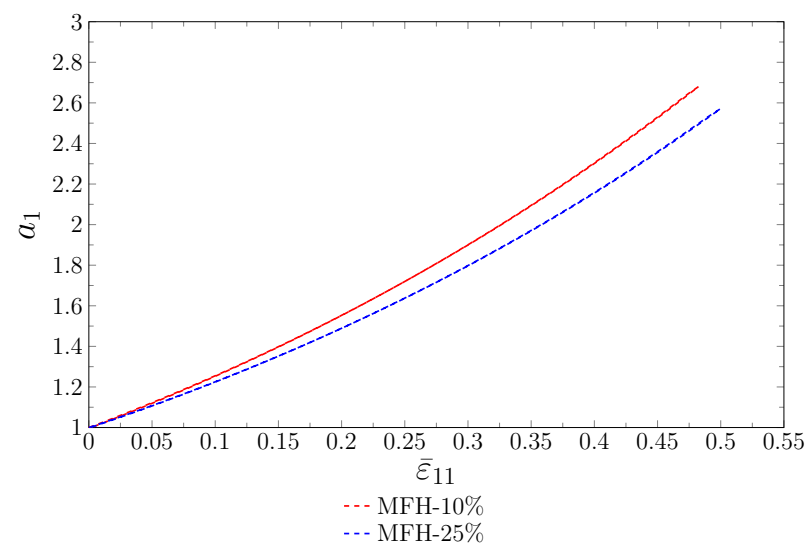

(c) Aspect ratio; tensile test

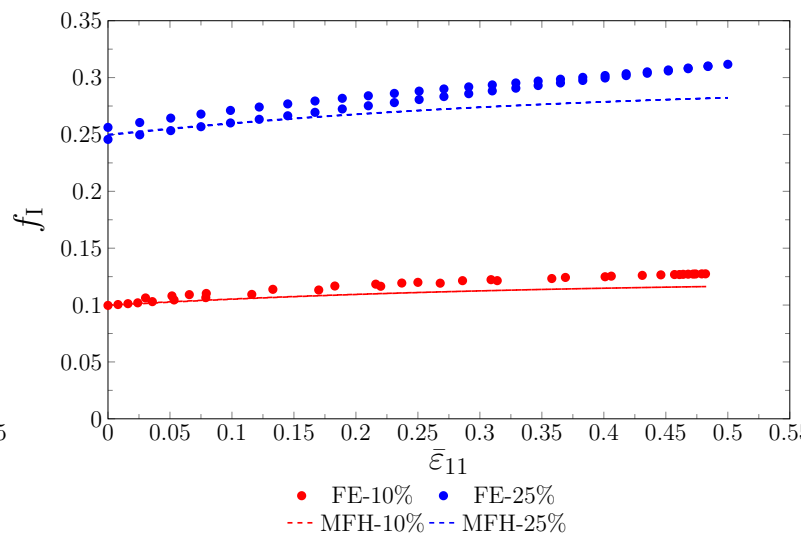

(b) Volume fraction; tensile test

Figure 9: Effective uni-axial tension compression cycle response of porous metallic materials containing $10 \%$ and $25 \%$ of initially spherical cavities: (a) normalized macro stress vs. macro strain, (b) porosity volume fraction vs. macro strain, (c) and porosity aspect ratio vs. macro strain. The response of the matrix without cavities is also provided for comparison. 

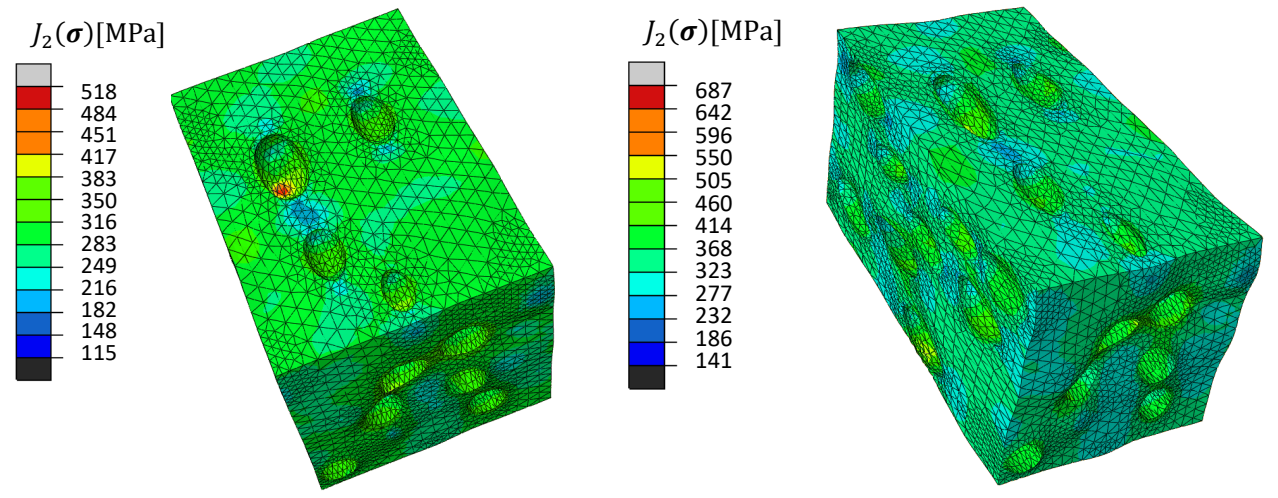

(a) $\bar{\epsilon}_{11}=0.25, \dot{\bar{\epsilon}}_{11}>0$

(b) $\bar{\epsilon}_{11}=0.48, \dot{\bar{\epsilon}}_{11}>0$
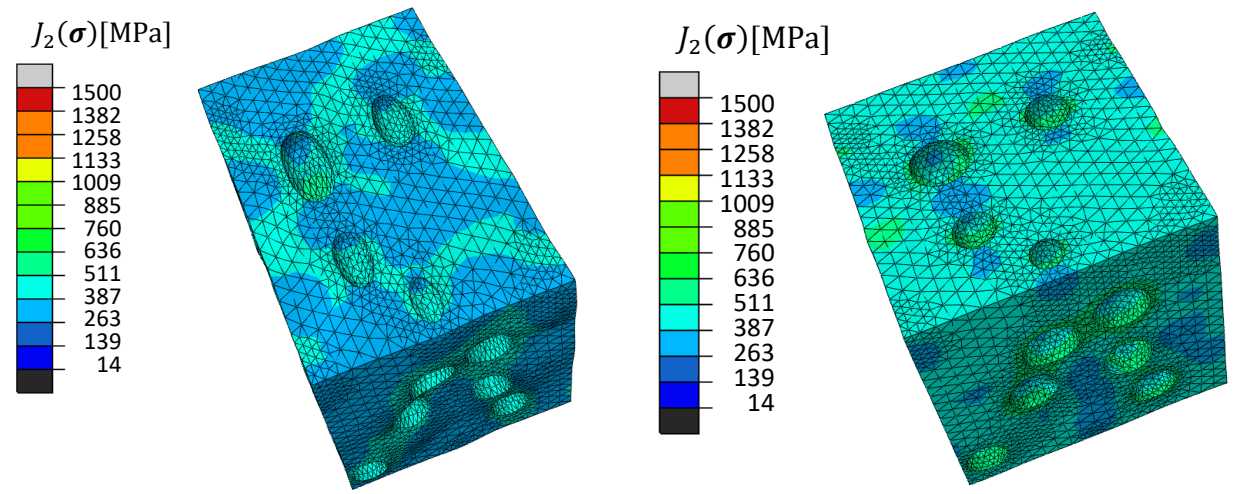

(c) $\bar{\epsilon}_{11}=0.33, \dot{\bar{\epsilon}}_{11}<0$

(d) $\bar{\epsilon}_{11}=0, \dot{\epsilon}_{11}<0$

Figure 10: Deformed RVEs of a porous metallic matrix containing 10\% initially spherical cavities and loaded under a unixial tension-compression cycle. 
The predictions of the effective response for a uni-axial tension compresson cyclic test are reported on Fig. 9(a) for the two initial cavity contents. The response of the matrix material without cavities is also presented to depict the influence of the holes. The figure shows that the MFH-incremental-secant model is able to reproduce fairly well the stress-strain curve. The evolutions of the porosity volume fraction predicted by the MFH scheme are reported in Fig. 9(b). It can be seen that the porosity is growing during uniaxial tension. As compared to the FE method, the variation of the porosity is underestimated with the MFH scheme. Finally, the evolution of the porosity aspect ratio predicted by the MFH is illustrated in Fig. 9(c): the aspect ratio is modified by a ratio of almost three as compared to the initial value. This value is in good qualitative agreement with the observed porosity shapes of the FE simulations reported in Fig. 10.

The porous material with $25 \%$ of initial cavity content is now tested under different loading conditions. The predictions of the effective response for a bi-axial tension test with $\bar{\varepsilon}_{11}=\bar{\varepsilon}_{22}$ and $\bar{\sigma}_{33}=0$ and for a shear test up to $\bar{F}_{12}=1$ are reported on Fig. 11(a) and on Fig. 11(d), respectively. The evolution of the unconstrained strain $\bar{\varepsilon}_{33}$ during the biaxial loading is illustrated in Fig. 11(b). The figures show that the MFH-incremental-secant model overestimates the porous material response, in particular in the case of biaxial loading. For macro hydrostatic loading conditions or for high macro stress triaxialities, the proposed incremental-secant formulation or the incremental-tangent one [27] lead to poor or erroneous, nonphysical predictions. Nevertheless, the porosity evolution is well predicted with the MFH as illustrated in Fig. 11(c). For a discussion and a recent proposal for spherical cavities embedded in a rigid-plastic matrix, we refer to [42].

\subsection{Dual-phase steel with ellipsoidal inclusions}

As a third illustration, we consider a dual-phase steel consisting of elastic inclusions (Martensite) dispersed in a ferrite-based matrix whose elasto-plastic hardening law follows Eq. (87). In this case, the Young's modulus of the inclusions phase is close to the one of the matrix phase. The following materials properties 


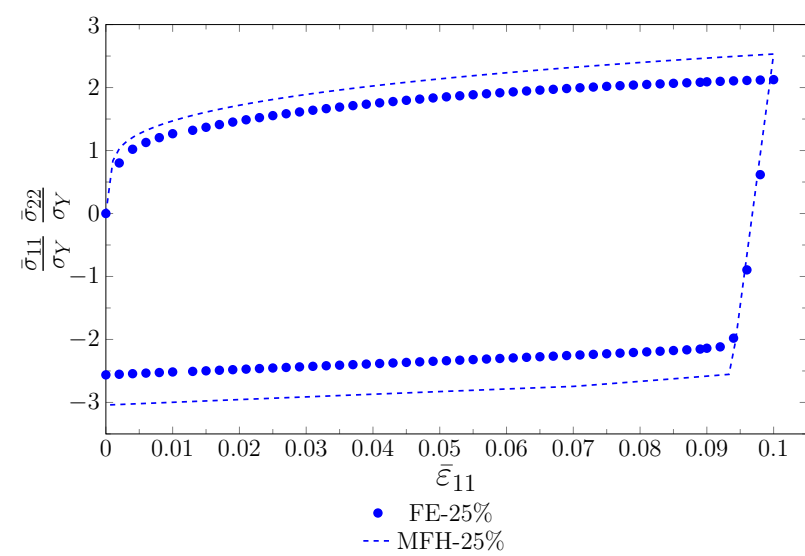

(a) Macro stresses; Biaxial loading

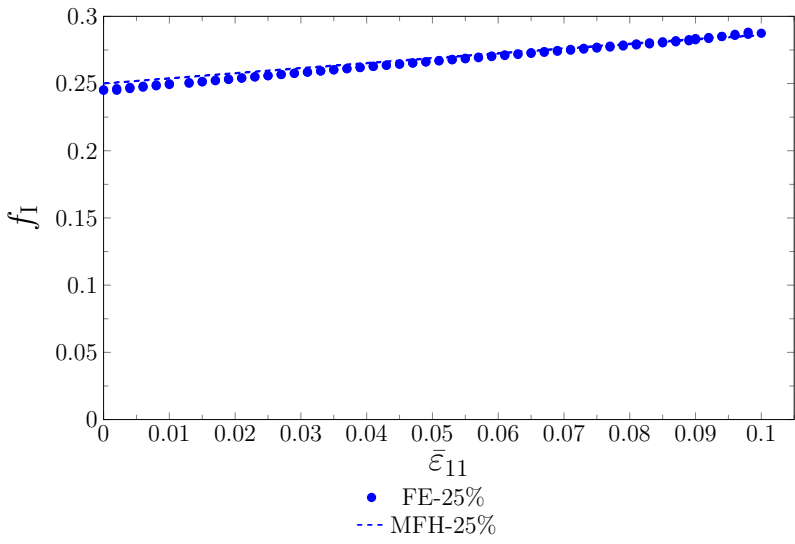

(c) Volume fraction; Biaxial loading

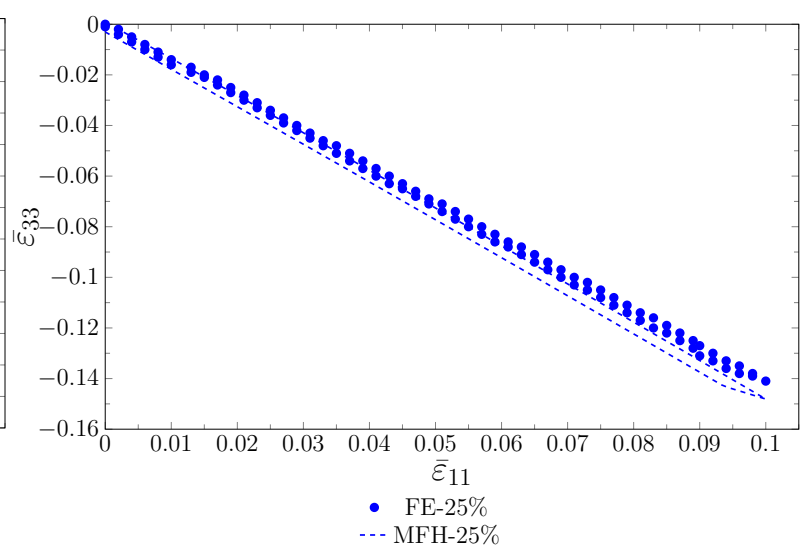

(b) Macro strains; Biaxial loading

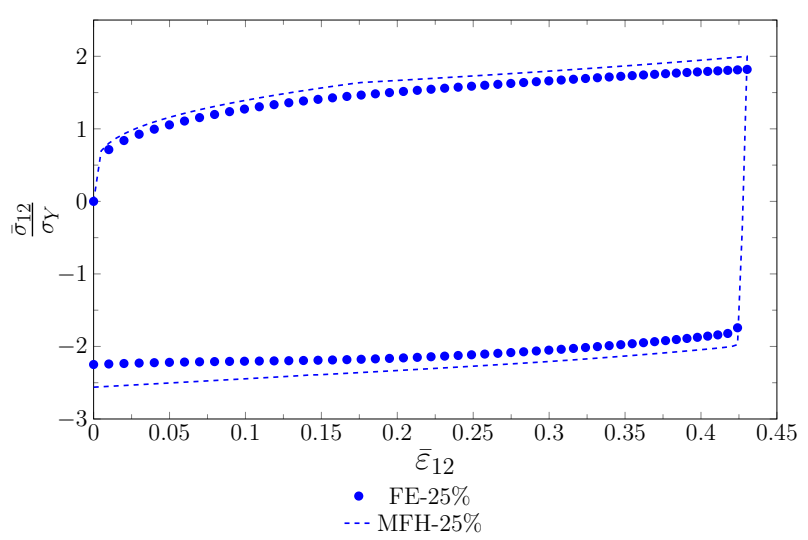

(d) Macro stresses; shear test

Figure 11: Effective bi-axial cyclic loading response, (a-c), and shear response, (d), of a porous metallic material containing $25 \%$ of initially spherical cavities. (a) and (d) Normalized macro stress vs. macro strain, (b) normalized unconstrained macro strain vs. constrained macro strains, and (c) porosity volume fraction vs. constrained macro strains. 


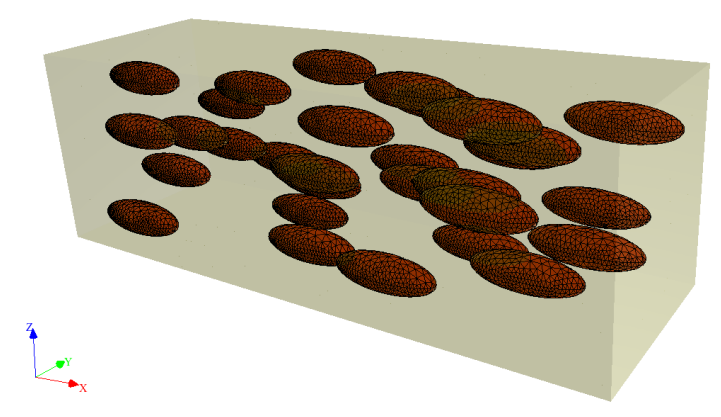

Figure 12: RVEs for FE analysis of a matrix material reinforced with $7.5 \%$ aligned ellipsoidal inclusions.

are thus:

- Inclusions: $E=250 \mathrm{GPa}, v=0.3$;

- Matrix: $E=220 \mathrm{GPa}, v=0.3, \sigma_{Y}=300 \mathrm{MPa}, h_{1}=1130 \mathrm{MPa}, n=0.31, \xi_{0}=0.11$, and $h_{2}=610 \mathrm{MPa}$.

This test aims at assessing the capability of the model to capture the effect of the morphology of the inclusions phase on the effective behavior of the composite material. As an illustration, we consider ellipsoidal inclusions with an aspect ratio of 3.

We consider an initial inclusions' volume fraction of $f_{0_{I}}=0.075$ as illustrated in Fig. 12 for 30 inclusions. Finite Element computations were conducted with the commercial solver Abaqus. The RVEs were meshed using 10-node tetrahedra (C3D10) and comprise about $2 \times 10^{5}$ elements.

The MFH predictions are compared to FE results in Fig. 13(a) for a uni-axial tension-compression cyclic loading along the ellipsoids direction and in Fig. 13(b) for a cyclic shearing up to $\bar{F}_{12}=1$ parallel to the ellipsoids, i.e. the ellipsoids are along the $x$-direction. The response of the matrix without reinforcement (i.e. $f_{0_{l}}=0$ ) is also considered in the uniaxial test to verify that the large deformation constitutive elasto-plastic formulation considered in the MFH is consistent with the one used by Abaqus. The phases responses for the two loading cases are illustrated in respectively Fig. 13(c) and Fig. 13(d). 


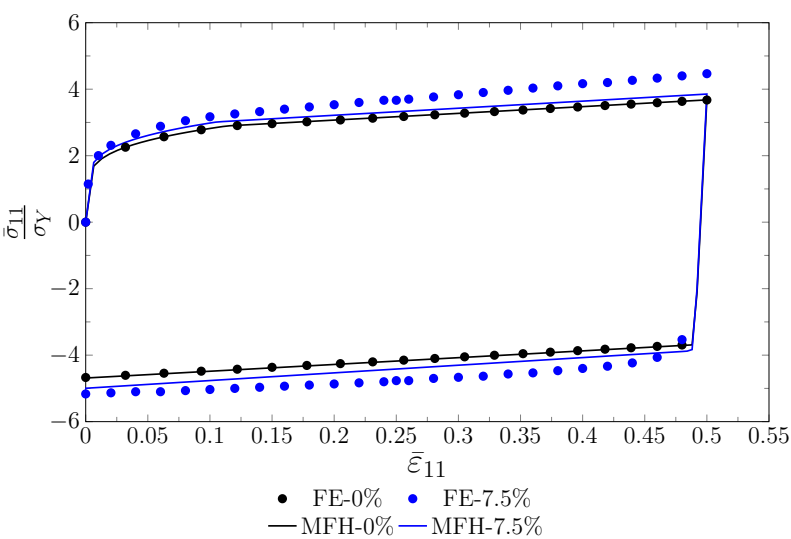

(a) Macro stresses; tensile test

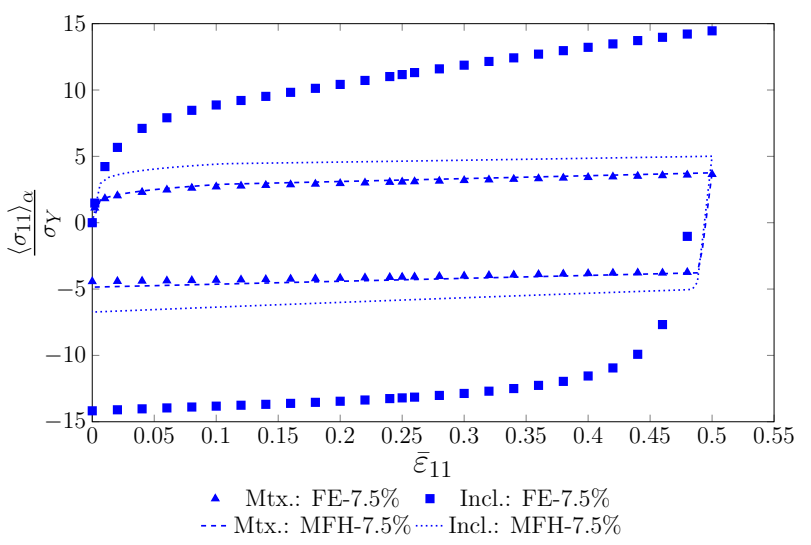

(c) Phase average stresses; tensile test

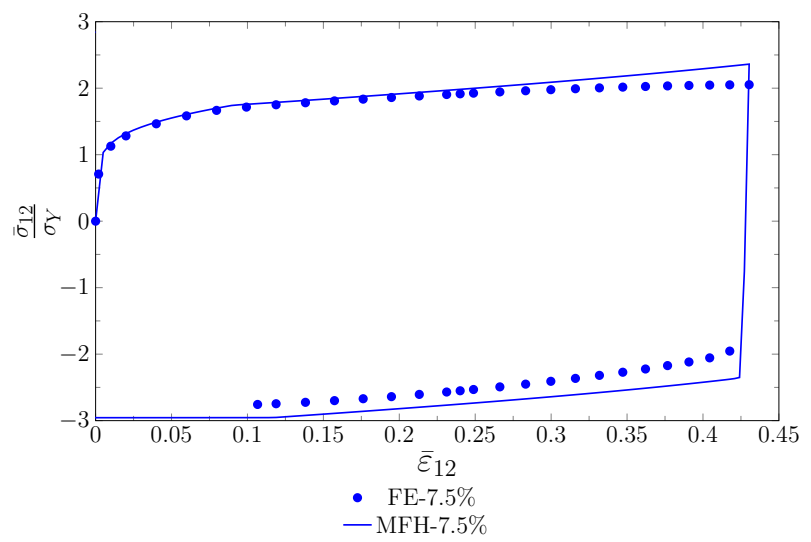

(b) Macro stresses; shear test

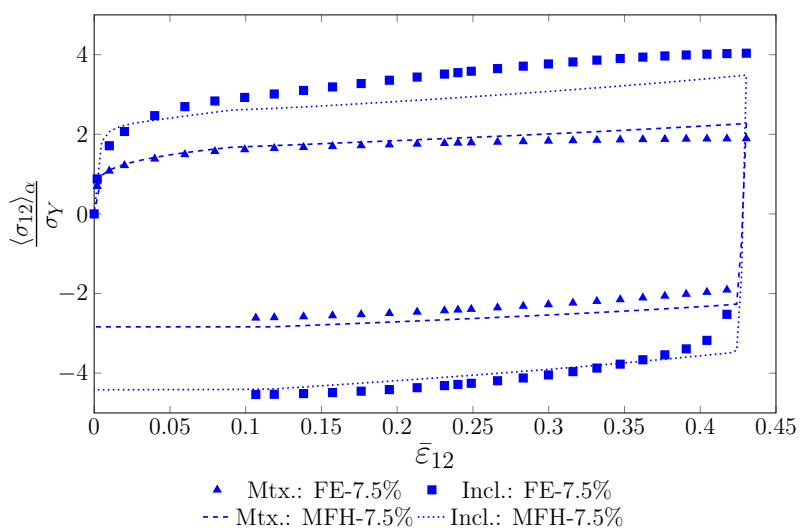

(d) Phase average stresses; shear test

Figure 13: Effective response, (a) and (b), and per-phase responses, (c) and (d), of dual-phase steel made of $7.5 \%$ of aligned ellipsoidal Martensite inclusions of aspect ratio 3 embedded in a ferritebased elasto-plastic matrix under (a) and (c) macro uni-axial tension compression cycle along the longitudinal direction, and (b) and (d) shearing parallel to the longitudinal direction. Normalized macro stress vs. macro strain. The response of the matrix is also provided for comparison in the tensite test. 
It can be seen that while the macro responses are reasonably predicted by the MFH scheme, see Figs. 13(a) and 13(b), the inclusion responses saturate in the $\mathrm{MFH}$, and are under-estimated as compared to the FE prediction, see Figs. 13(c) and 13(d). This behavior results from the fact that the matrix exhibits a nearly perfectly plastic behavior, in which case the residual-incremental-secant operator should be used in both phases in combination with second statistical moments.

\section{Conclusions}

We proposed a finite strain incremental-secant MFH formulation which presents several original features with respect to the existing literature. For hyper-elasticplastic constituents, the local models follow the framework of the thermodynamics of irreversible processes and are based on two main assumptions: (i) a multiplicative decomposition of the deformation gradient into elastic and inelastic parts; and (ii) a hyper-elastic relation between the Kirchhoff stress and the elastic left CauchyGreen strain tensors. At first, an incremental-secant formulation was formulated in the material constituents by considering a virtual unloading of the material phase upon which a radial return algorithm was formulated following a secant approach. In particular it was shown that by considering elastic logarithmic strains in the hyper-elastic approach, the finite strains form of the incremental-secant approach is written in a similar way as in small deformation. The incremental-secant MFH scheme previously developed in infinitesimal strain could thus be directly adapted to the finite strains case. A computational algorithm was then developed for a finite strain version of the Mori-Tanaka MFH model and numerically implemented.

To discuss the accuracy of the presented approach, different micro-structure systems were numerically tested: elasto-plastic matrix with elastic inclusions and porous elasto-plastic matrix. Initially spherical or ellipsoidal inclusions were considered as well as different loading conditions: uni-axial, bi-axial, and shearing loading-unloading cycles. Different values of the initial volume fraction of solid inclusions and of the porosity were also considered. The MFH predictions were 
compared against direct full-field FE simulations on RVEs and found to be accurate in the composite material response, except in the case in which the matrix elastoplastic behavior became perfectly plastic.. In the case of porous materials, the MFH predictions were shown to be able to capture the material behavior as well as the porosity and cavity shape evolutions.

The MFH method proposed in this paper was based on first statistical moments, i.e. only the mean values (volume averages) of the per-phase deformation gradient are used to compute an approximation of the mean nominal stresses in the phases. More accurate predictions, in particular in the case of a perfectly plastic behavior, are expected by enriching the approach with the second statistical moments, which would enable to measure the variance of the micro fields. Introducing these second statistical moments has been achieved in small deformations in the case of history dependent materials in [43], [44], [45], [46], [47] and [26], each based on a different approach, including using the residual-incremental-secant MFH [44, 26], but has yet to be done in finite strains.

Finally, materials can exhibit strain localization, either because of the large deformation, e.g. [48], [49], because of the introduction of a damage model, e.g. [50], or again because of the micro-structure (porous or composite materials), e.g. [51] and [52]. One important consequence of localization is the loss of uniqueness. Although MFH homogenization schemes have been coupled with a non-local formulation to avoid the loss of solution uniqueness, this has only been achieved in the context of infinitesimal strain [25, 4]. The incremental-secant formulation was able to capture with high accuracy the unloading of the inclusions phase during the strain softening of the matrix material in [4]. It is thus expected that the presented homogenization approach can be extended to account for strain softening in large deformations.

\section{References}

[1] P. Kanouté, D. P. Boso, J. L. Chaboche, B. a. Schrefler, Multiscale methods for composites: A review, Archives of Computational Methods in 
Engineering 16 (1) (2009) 31-75, ISSN 11343060, doi: \bibinfo\{doi\}\{10.1007/ s11831-008-9028-8\}.

[2] K. Matous, M. G. Geers, V. G. Kouznetsova, A. Gillman, A review of predictive nonlinear theories for multiscale modeling of heterogeneous materials, Journal of Computational Physics 330 (2017) 192 - 220, ISSN 0021-9991, doi: \bibinfo\{doi\}\{10.1016/j.jcp.2016.10.070\}.

[3] P. Ponte Castañeda, J. Willis, The effect of spatial distribution on the effective behavior of composite materials and cracked media, Journal of the Mechanics and Physics of Solids 43 (12) (1995) 1919 - 1951, ISSN 0022-5096, doi:〈bibinfo\{doi\}\{https://doi.org/10.1016/0022-5096(95)00058-Q\}, URL http: //www.sciencedirect.com/science/article/pii/002250969500058Q.

[4] L. Wu, L. Noels, L. Adam, I. Doghri, An implicit-gradient-enhanced incremental-secant mean-field homogenization scheme for elasto-plastic composites with damage, International Journal of Solids and Structures 50 (24) (2013) 3843 - 3860, ISSN 0020-7683, doi:〈bibinfo\{doi\}\{http:// dx.doi.org/10.1016/j.jjsolstr.2013.07.022\}, URL http://www. sciencedirect. com/science/article/pii/S0020768313003028.

[5] J. D. Eshelby, The Determination of the Elastic Field of an Ellipsoidal Inclusion, and Related Problems, Proceedings of the Royal Society A: Mathematical, Physical and Engineering Sciences 241 (1226) (1957) 376-396, ISSN 1364-5021, doi: \bibinfo\{doi\}\{10.1098/rspa.1957.0133\}.

[6] S. Nemat-Nasser, M. Hori, Micromechanics: overall properties of heterogeneous materials, Elsevier North-Holland, 1999.

[7] D. R. S. Talbot, J. R. Willis, Variational Principles for Inhomogeneous Nonlinear Media, IMA Journal of Applied Mathematics 35 (1) (1985) 39-54, doi: |bibinfo\{doi\}\{10.1093/imamat/35.1.39\}, URL +http://dx. doi . org/10 . 1093/ imamat/35.1.39. 
[8] D. R. S. Talbot, J. R. Willis, Bounds and Self-Consistent Estimates for the Overall Properties of Nonlinear Composites, IMA Journal of Applied Mathematics 39 (3) (1987) 215-240, doi:〈bibinfo\{doi\}\{10.1093/imamat/39.3.215\}.

[9] P. Ponte Castañeda, The effective mechanical properties of nonlinear isotropic composites, Journal of the Mechanics and Physics of Solids 39 (1) (1991) 45 - 71, ISSN 0022-5096, doi:|bibinfo\{doi\}\{http://dx.doi. org/10.1016/0022-5096(91)90030-R\}, URL http://www. sciencedirect.com/ science/article/pii/002250969190030R.

[10] P. Ponte Castañeda, A New Variational Principle and its Application to Nonlinear Heterogeneous Systems, SIAM Journal on Applied Mathematics 52 (5) (1992) 1321-1341, ISSN 00361399.

[11] D. Talbot, J. Willis, Some simple explicit bounds for the overall behaviour of nonlinear composites, International Journal of Solids and Structures 29 (14-15) (1992) 1981 - 1987, ISSN 0020-7683, doi: \bibinfo\{doi\}\{DOI:10.1016/ 0020-7683(92)90188-Y\}.

[12] A. Molinari, F. El Houdaigui, L. Tóth, Validation of the tangent formulation for the solution of the non-linear Eshelby inclusion problem, International Journal of Plasticity 20 (2) (2004) 291 - 307, ISSN 07496419, doi: \bibinfo\{doi\}\{10.1016/S0749-6419(03)00038-X\}, URL http://www . sciencedirect.com/science/article/pii/S074964190300038X.

[13] M. Berveiller, A. Zaoui, An extension of the self-consistent scheme to plastically-flowing polycrystals, Journal of the Mechanics and Physics of Solids 26 (5-6) (1978) 325 - 344, ISSN 0022-5096, doi:|bibinfo\{doi\}\{DOI: 10.1016/0022-5096(78)90003-0\}.

[14] R. Hill, Continuum micro-mechanics of elastoplastic polycrystals, J. Mech. Phys. Solids 13 (1965) 89-101. 
[15] H. E. Pettermann, A. F. Plankensteiner, H. J. Böhm, F. G. Rammerstorfer, A thermo-elasto-plastic constitutive law for inhomogeneous materials based on an incremental Mori-Tanaka approach, Computers \& Structures 71 (2) (1999) 197 - 214, ISSN 0045-7949, doi:\bibinfo\{doi\}\{DOI:10.1016/ S0045-7949(98)00208-9\}.

[16] I. Doghri, A. Ouaar, Homogenization of two-phase elasto-plastic composite materials and structures: Study of tangent operators, cyclic plasticity and numerical algorithms, International Journal of Solids and Structures 40 (7) (2003) 1681 - 1712, ISSN 0020-7683, doi:\bibinfo\{doi\}\{DOI: 10.1016/S0020-7683(03)00013-1\}.

[17] I. Doghri, L. Tinel, Micromechanical modeling and computation of elasto-plastic materials reinforced with distributed-orientation fibers, International Journal of Plasticity 21 (10) (2005) 1919 - 1940, ISSN 0749-6419, doi:\bibinfo\{doi\}\{10.1016/j.ijplas.2004.09.003\}, URL http://www . sciencedirect.com/science/article/pii/S0749641904001706.

[18] O. Pierard, I. Doghri, Study of various estimates of the macroscopic tangent operator in the incremental homogenization of elasto-plastic composites, International Journal for Multiscale Computational Engineering 4 (2006) $521-543$.

[19] A. Molinari, G. Canova, S. Ahzi, A self consistent approach of the large deformation polycrystal viscoplasticity, Acta Metallurgica 35 (12) (1987) 29832994.

[20] R. Masson, M. Bornert, P. Suquet, A. Zaoui, An affine formulation for the prediction of the effective properties of nonlinear composites and polycrystals, J. Mech. Phys. Solids 48 (2000) 1203-1227.

[21] A. Zaoui, R. Masson, Modelling Stress-Dependent Transformation Strains of Heterogeneous Materials, in: Y. A. Bahei-El-Din, G. J. Dvorak, G. M. L. 
Gladwell (Eds.), IUTAM Symposium on Transformation Problems in Composite and Active Materials, vol. 60 of Solid Mechanics and Its Applications, Springer Netherlands, ISBN 978-0-306-46935-0, 3-15, doi:〈bibinfo\{doi\}\{10. 1007/0-306-46935-9_1\}, 2002.

[22] S. Mercier, A. Molinari, Homogenization of elasticviscoplastic heterogeneous materials: Self-consistent and Mori-Tanaka schemes, International Journal of Plasticity 25 (6) (2009) 1024 - 1048, ISSN 0749-6419, doi:|bibinfo\{doi\}\{10. 1016/j.ijplas.2008.08.006\}, URL http://www.sciencedirect.com/science/ article/pii/S0749641908001290.

[23] J. Chaboche, P. Kanouté, A. Roos, On the capabilities of mean-field approaches for the description of plasticity in metal matrix composites, International Journal of Plasticity 21 (7) (2005) 1409 - 1434, ISSN 0749-6419, doi: bibinfo\{doi\}\{DOI:10.1016/j.ijplas.2004.07.001\}.

[24] L. Wu, L. Noels, L. Adam, I. Doghri, A combined incremental-secant meanfield homogenization scheme with per-phase residual strains for elastoplastic composites, International Journal of Plasticity 51 (2013) 80-102, ISSN 07496419, doi: \bibinfo\{doi\}\{10.1016/j.ijplas.2013.06.006\}.

[25] L. Wu, L. Noels, L. Adam, I. Doghri, A multiscale mean-field homogenization method for fiber-reinforced composites with gradient-enhanced damage models, Computer Methods in Applied Mechanics and Engineering 233-236 (2012) 164 - 179, ISSN 0045-7825, doi:|bibinfo\{doi\}\{https://doi.org/ 10.1016/j.cma.2012.04.011\}, URL http://www. sciencedirect. com/science/ article/pii/S0045782512001314.

[26] L. Wu, L. Adam, I. Doghri, L. Noels, An incremental-secant meanfield homogenization method with second statistical moments for elasto-visco-plastic composite materials, Mechanics of Materials 114 (2017) 180 - 200, ISSN 0167-6636, doi:〈bibinfo\{doi\}\{https://doi.org/10.1016/ 
j.mechmat.2017.08.006\}, URL http://www.sciencedirect.com/science/ article/pii/S0167663617300698.

[27] I. Doghri, M. E. Ghezal, L. Adam, Finite strain mean-field homogenization of composite materials with hyperelastic-plastic constituents, International Journal of Plasticity 81 (2016) 40 - 62, ISSN 0749-6419, doi: |bibinfo\{doi\}\{https://doi.org/10.1016/j.ijplas.2016.01.009\}, URL http://www . sciencedirect.com/science/article/pii/S074964191600019X.

[28] P. Ponte Castañeda, E. Tiberio, Second-order homogenization method in finite elasticity and applications to black-filled elastomers, Journal of the Mechanics and Physics of Solids 48 (6) (2000) 1389-1411, ISSN 00225096, doi:〈bibinfo\{doi\} $\{10.1016 /$ S0022-5096(99)00087-3\}.

[29] P. Ponte Castañeda, P. Suquet, Nonlinear Composites and Microstructure Evolution, in: H. Aref, J. Phillips (Eds.), Mechanics for a New Mellennium, Springer Netherlands, ISBN 978-0-7923-7156-4, 253-274, doi:|bibinfo\{doi\}\{10.1007/0-306-46956-1-17\}, URL http://dx.doi.org/10. $1007 / 0-306-46956-1-17,2002$.

[30] M. I. Idiart, K. Danas, P. Ponte Castañeda, Second-order theory for nonlinear composites and application to isotropic constituents, Comptes Rendus Mecanique 334 (10) (2006) 575-581, ISSN 16310721, doi: \bibinfo\{doi\}\{10.1016/ j.crme.2006.06.006\}.

[31] D. Song, M. Agoras, P. Ponte Castañeda, The evolution of pore shape and orientation in plastically deforming metals: Implications for macroscopic response and shear localization, Mechanics of Materials 90 (2015) 47 - 68, ISSN 0167-6636, doi:〈bibinfo\{doi\}\{https://doi.org/10.1016/ j.mechmat.2015.01.011\}, URL http://www.sciencedirect.com/science/ article/pii/S0167663615000186, proceedings of the IUTAM Symposium on Micromechanics of Defects in Solids. 
[32] E. H. Lee, Elastic-Plastic Deformation at Finite Strains, Journal of Applied Mechanics 36 (1) (1969) 1-6, doi: \bibinfo\{doi\}\{http://dx.doi.org/10.1115/1. $3564580\}$.

[33] R. Radovitzky, M. Ortiz, Error estimation and adaptive meshing in strongly nonlinear dynamic problems, Computer Methods in Applied Mechanics and Engineering 172 (1) (1999) 203-240, ISSN 0045-7825, doi: \bibinfo\{doi\}\{https:// doi.org/10.1016/S0045-7825(98)00230-8\}, URL http://www. sciencedirect. com/science/article/pii/S0045782598002308.

[34] M. Ortiz, L. Stainier, The variational formulation of viscoplastic constitutive updates, Computer Methods in Applied Mechanics and Engineering 171 (3) (1999) 419 - 444, ISSN 0045-7825, doi:〈bibinfo\{doi\}\{https://doi. org/10.1016/S0045-7825(98)00219-9\}, URL http: //www . sciencedirect . com/ science/article/pii/S0045782598002199.

[35] A. Mielke, Energetic formulation of multiplicative elasto-plasticity using dissipation distances, Continuum Mechanics and Thermodynamics 15 (4) (2003) 351-382, ISSN 1432-0959, doi: \bibinfo $\{$ doi $\{10.1007 /$ s00161-003-0120-x\}, URL https://doi.org/10.1007/s00161-003-0120-x.

[36] A. Mainik, A. Mielke, Global Existence for Rate-Independent Gradient Plasticity at Finite Strain, Journal of Nonlinear Science 19 (3) (2009) 221-248, ISSN 1432-1467, doi:〈bibinfo\{doi\}\{10.1007/s00332-008-9033-y\}, URL https: //doi.org/10.1007/s00332-008-9033-y.

[37] A. Mielke, R. Rossi, G. Savaré, Global Existence Results for Viscoplasticity at Finite Strain, Archive for Rational Mechanics and Analysis 227 (1) (2018) 423-475, ISSN 1432-0673, doi: \bibinfo\{doi\}\{10.1007/s00205-017-1164-6\}, URL https://doi .org/10.1007/s00205-017-1164-6.

[38] J. Simo, Algorithms for static and dynamic multiplicative plasticity that preserve the classical return mapping schemes of the infinitesimal theory, Com- 
puter Methods in Applied Mechanics and Engineering 99 (1) (1992) 61-112, ISSN 00457825, doi:|bibinfo\{doi\}\{10.1016/0045-7825(92)90123-2\}.

[39] I. Doghri, Mechanics of deformable solids: linear and nonlinear, analytical and computational aspects., Springer, 2000.

[40] J. Simo, T. Hughes, Computational Inelasticity, Interdisciplinary Applied Mathematics, Springer New York, ISBN 9780387975207, URL https:// books . google . be/books?id=ftL2AJL80PYC, 2000.

[41] S. Nemat-Nasser, Averaging theorems in finite deformation plasticity, Mechanics of Materials 31 (1999) 493-523, ISSN 01676636, doi:〈bibinfo\{doi\} \{10.1016/S0167-6636(98)00073-8\}, URL http://www.sciencedirect.com/ science/article/pii/S0167663698000738.

[42] M. I. El Ghezal, I. Doghri, Porous plasticity: predictive second moment homogenization models coupled with Gurson's single cavity stressstrain solution, International Journal of Plasticity 108 (2018) 201-221, doi: \bibinfo\{doi\}\{https://doi.org/10.1016/j.ijplas.2018.05.006\}.

[43] I. Doghri, L. Brassart, L. Adam, J. S. Gérard, A second-moment incremental formulation for the mean-field homogenization of elasto-plastic composites, International Journal of Plasticity 27 (3) (2011) 352-371, ISSN 07496419, doi: \bibinfo\{doi\}\{10.1016/j.ijplas.2010.06.004\}, URL http://dx . doi .org/10. 1016/j.ijplas.2010.06.004.

[44] L. Wu, I. Doghri, L. Noels, An incremental-secant mean-field homogenization method with second statistical moments for elasto-plastic composite materials, Philosophical Magazine 95 (28-30) (2015) 3348-3384, doi: |bibinfo\{doi\}\{10.1080/14786435.2015.1087653\}, URL http://dx . doi . org/10 . $1080 / 14786435.2015 .1087653$.

[45] L. Brassart, L. Stainier, I. Doghri, L. Delannay, A variational formulation for the incremental homogenization of elasto-plastic composites, Journal of the 
Mechanics and Physics of Solids 59 (12) (2011) 2455-2475, ISSN 00225096, doi:〈bibinfo\{doi\}\{10.1016/j.jmps.2011.09.004\}, URL http://dx.doi.org/10. 1016/j . jmps. 2011.09.004.

[46] L. Brassart, L. Stainier, I. Doghri, L. Delannay, Homogenization of elasto(visco) plastic composites based on an incremental variational principle, International Journal of Plasticity 36 (2012) 86-112, ISSN 07496419, doi:|bibinfo\{doi\}\{10.1016/j.ijplas.2012.03.010\}, URL http://dx. doi .org/10. 1016/j.ijplas.2012.03.010.

[47] N. Lahellec, P. Suquet, Effective response and field statistics in elastoplastic and elasto-viscoplastic composites under radial and non-radial loadings, International Journal of Plasticity 42 (2013) 1-30, ISSN 07496419, doi: |bibinfo\{doi\}\{10.1016/j.ijplas.2012.09.005\}.

[48] R. Hill, Acceleration waves in solids, Journal of the Mechanics and Physics of Solids 10 (1) (1962) 1-16, ISSN 00225096, doi:〈bibinfo\{doi\}\{10.1016/ 0022-5096(62)90024-8\}.

[49] J. R. Rice, The localization of plastic deformation, 14th International Congress on Theoratical and Applied Mechanics (1976) 207-220doi: \bibinfo\{doi\}\{10.1. $1.160 .6740\}$.

[50] I. Doghri, R. Billardon, Investigation of localization due to damage in elasto-plastic materials, Mechanics of Materials 19 (2-3) (1995) 129-149, ISSN 01676636, doi:〈bibinfo\{doi\}\{10.1016/0167-6636(94)00011-5\}, URL http: //www.sciencedirect.com/science/article/pii/0167663694000115.

[51] K. Danas, P. Ponte Castañeda, Influence of the Lode parameter and the stress triaxiality on the failure of elasto-plastic porous materials, International Journal of Solids and Structures 49 (11-12) (2012) 1325-1342, ISSN 00207683, doi:|bibinfo\{doi\}\{10.1016/j.ijsolstr.2012.02.006\}, URL http://dx. doi.org/10.1016/j.ijsolstr.2012.02.006. 
[52] V. Tvergaard, Bifurcation into a localized mode from non-uniform periodic deformations around a periodic pattern of voids, Journal of the Mechanics and Physics of Solids 69 (2014) 112 - 122, ISSN 0022-5096, doi:〈bibinfo\{doi\}\{http://dx.doi.org/10.1016/j.jmps.2014.05.002\}, URL http:// www.sciencedirect.com/science/article/pii/S0022509614000854.

\section{Appendix A. Hyperelastic-plastic constitutive equations}

The definition of the Lie derivative $\boldsymbol{b}^{\underline{e}} \equiv \boldsymbol{F} \cdot \frac{\mathrm{d}}{\mathrm{d} t}\left(\boldsymbol{C}^{p-1}\right) \cdot \boldsymbol{F}^{T}$ applied to Eq. (7) yields relation (10), which also reads

$$
\dot{b}^{e}=\stackrel{L}{e}+\boldsymbol{l} \cdot \boldsymbol{b}^{e}+\boldsymbol{b}^{e} \cdot \boldsymbol{l}^{T}
$$

with the velocity gradient $\boldsymbol{l}=\dot{\boldsymbol{F}} \cdot \boldsymbol{F}^{-1}$. Considering a specific free energy per unit reference volume $\psi\left(\boldsymbol{b}^{e}, \xi\right)$ as an isotropic function of $\boldsymbol{b}^{e}$ and of an internal

scalar variable $\xi$ implies that $\frac{\partial \psi}{\partial \boldsymbol{b}^{e}}$ and $\boldsymbol{b}^{e}$ commute. Therefore, using Eq. (A.1), the following chainrule applies

$$
\begin{aligned}
\dot{\psi} & =\frac{\partial \psi}{\partial \boldsymbol{b}^{e}}: \dot{\boldsymbol{b}}^{e}+\frac{\partial \psi}{\partial \xi} \dot{\xi}=\frac{\partial \psi}{\partial \boldsymbol{b}^{e}}:\left(\boldsymbol{l} \cdot \boldsymbol{b}^{e}+\boldsymbol{b}^{e} \cdot \boldsymbol{l}\right)+\frac{\partial \psi}{\partial \boldsymbol{b}^{e}}: \boldsymbol{b}^{e}+\frac{\partial \psi}{\partial \xi} \dot{\xi} \\
& =\left(\frac{\partial \psi}{\partial \boldsymbol{b}^{e}} \cdot \boldsymbol{b}^{e}\right):\left(\boldsymbol{l}+\boldsymbol{l}^{T}\right)+\left(\frac{\partial \psi}{\partial \boldsymbol{b}^{e}} \cdot \boldsymbol{b}^{e}\right):\left(\boldsymbol{b}^{L} \cdot \boldsymbol{b}^{e-1}\right)+\frac{\partial \psi}{\partial \xi} \dot{\xi}
\end{aligned}
$$

The Clausius-Duhem dissipation defined as

$$
\mathcal{D} \equiv \tau: \frac{1}{2}\left(l+l^{T}\right)-\dot{\psi} \geq 0,
$$

thus becomes

$$
\mathcal{D} \equiv\left(\boldsymbol{\tau}-2 \frac{\partial \psi}{\partial \boldsymbol{b}^{e}} \cdot \boldsymbol{b}^{e}\right): \frac{1}{2}\left(\boldsymbol{l}+\boldsymbol{l}^{T}\right)+\left(2 \frac{\partial \psi}{\partial \boldsymbol{b}^{e}} \cdot \boldsymbol{b}^{e}\right):\left(-\frac{1}{2} \boldsymbol{b}^{e} \cdot \boldsymbol{b}^{e-1}\right)-\frac{\partial \psi}{\partial \xi} \dot{\xi} \geq 0
$$

This relation yields the Kirchhoff stress equation of state $\tau=2 \frac{\partial \psi}{\partial \boldsymbol{b}^{e}} \cdot \boldsymbol{b}^{e}$ and the definition of the thermodynamic force associated with $\xi$, i.e. $q \equiv \frac{\partial \psi}{\partial \xi}$. Using these 
relations, the Clausius-Duhem inequality (A.3) is rewritten in the reduced form

$$
\mathcal{D}=\boldsymbol{\tau}:\left(-\frac{1}{2} \boldsymbol{b}^{e} \cdot \boldsymbol{b}^{e-1}\right)-q \dot{\xi} \geq 0
$$

\section{Appendix B. Evaluation of the residual stress vector and material operator}

In this section, we neglect the derivatives of rotation tensors $\tilde{\boldsymbol{R}}_{n+1_{\alpha}}^{n}$ since it did not affect the convergence of the homogenization scheme.

Appendix B.1. Derivation of the incremental-secant operator

We first consider the case of the residual-incremental-secant operator (59). The evaluation of $\frac{\partial C^{S r}}{\partial \varepsilon}$ used in the MFH scheme follows from

$$
\frac{\partial C^{S r}}{\partial \varepsilon}=\frac{\partial}{\partial \Delta \varepsilon^{r}}\left(3 k^{S r} \mathcal{I}^{v o l}+2 G^{S r} \mathcal{I}^{d e v}\right): \frac{\partial \Delta \varepsilon^{r}}{\partial \varepsilon}=2 \mathcal{I}^{d e v} \otimes \frac{\partial G^{S r}}{\partial \Delta \varepsilon^{r}} .
$$

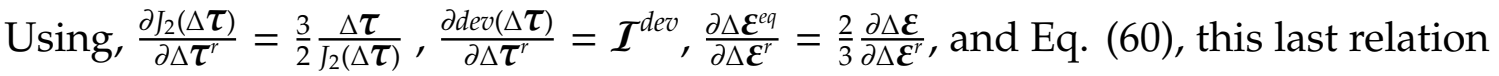
becomes

$$
\frac{\partial C^{S r}}{\partial \varepsilon}=2 I^{d e v} \otimes\left[\frac{1}{6 G^{S r}\left(\Delta \varepsilon^{e q}\right)^{2}} \operatorname{dev}(\Delta \tau): C^{a l g}-\frac{2}{3} G^{S r} \frac{\operatorname{dev}\left(\Delta \varepsilon^{r}\right)}{\left(\Delta \varepsilon^{e q}\right)^{2}}\right]
$$

where $\Delta \varepsilon^{e q}=\sqrt{\frac{2}{3} \operatorname{dev}\left(\Delta \varepsilon_{n+1_{\alpha}}^{r}\right): \operatorname{dev}\left(\Delta \varepsilon_{n+1_{\alpha}}^{r}\right)}$. In Eq. (B.2), $C^{a l g}$ is the derivative of the stress increment with respect to the strain increment, which is obtained from the constitutive law of the material. Due to the modification of the return mapping algorithm, this expression is slightly changed compared to the usual one and reads

$$
C^{a l g}=C^{e l}-\frac{\left(2 G^{e l}\right)^{2}}{h} N \otimes N-\frac{\left(2 G^{e l}\right)^{2} \Delta \gamma}{\left(\tau_{n+1}^{t r}-\tau_{n}^{r e s}\right)^{e q}}\left(\frac{2}{3} I^{d e v}-N \otimes N\right),
$$

with $h=\frac{1}{3} N:\left(\frac{3}{2} \frac{\operatorname{dev}\left(\boldsymbol{\tau}_{n+1}\right)}{J_{2}(\boldsymbol{\tau})}\right)^{-1} \frac{\partial \eta}{\partial \xi}+3 G^{e l}$.

The case of the zero-incremental-secant operator (63) can be easily deduced by 
considering Eq. (64) instead of (60) when evaluating Eq. (B.2), leading to

$$
\frac{\partial C^{S 0}}{\partial \boldsymbol{\varepsilon}}=3 \frac{k^{e l}-k^{S 0}}{\operatorname{tr}\left(\Delta \varepsilon_{n+1_{\alpha}}^{r}\right)} I^{v o l} \times I+2 \mathcal{I}^{d e v} \otimes\left[\frac{1}{6 G^{S 0}\left(\Delta \varepsilon^{e q}\right)^{2}} \operatorname{dev}(\Delta \tau): C^{a l g}-\frac{2}{3} G^{S 0} \frac{\operatorname{dev}\left(\Delta \boldsymbol{\varepsilon}^{r}\right)}{\left(\Delta \varepsilon^{e q}\right)^{2}}\right],
$$

and the classical normal direction (62) when evaluating $C^{a l g}$, leading to

$$
C^{a l g}=C^{e l}-\frac{\left(2 G^{e l}\right)^{2}}{h} N \otimes N-\frac{\left(2 G^{e l}\right)^{2} \Delta \gamma}{\left(\tau_{n+1}^{t r}\right)^{e q}}\left(\frac{2}{3} I^{d e v}-N \otimes N\right) .
$$

Appendix B.2. Residual stress vector and Jacobian evaluation

The equation to be satisfied at the end of the MFH procedure is Eq. (67). The averaging rule is applied in this work to the residual strains increments as:

$$
\Delta \bar{\varepsilon}_{n+1}^{r}=f_{M} \Delta \varepsilon_{n+1_{M}}^{r}+f_{I} \Delta \varepsilon_{n+1_{I}}^{r} .
$$

Multiplying Eq. (B.6) by $\boldsymbol{B}^{\epsilon}\left(I, \boldsymbol{C}_{0}^{S}, \boldsymbol{C}_{I}^{S}\right)$ and using Eq. (67) lead to:

$$
f_{M} \Delta \boldsymbol{\varepsilon}_{n+1_{I}}^{r}+f_{I} \boldsymbol{B}^{\epsilon}\left(I, \boldsymbol{C}_{0}^{S}, \boldsymbol{C}_{I}^{S}\right): \Delta \boldsymbol{\varepsilon}_{n+1_{I}}^{r}=\boldsymbol{B}^{\epsilon}\left(I, \boldsymbol{C}_{0}^{S}, \boldsymbol{C}_{I}^{S}\right): \Delta \overline{\boldsymbol{\varepsilon}}_{n+1}^{r} .
$$

With the M-T assumption, the strain concentration tensor follows from Eq. (68), and Eq. (B.7) reads

$$
\Delta \boldsymbol{\varepsilon}_{n+1_{I}}^{r}+f_{M} \mathcal{S}:\left[\left(\mathcal{C}_{M}^{S}\right)^{-1}: \mathcal{C}_{I}^{S}-\mathcal{I}\right]: \Delta \boldsymbol{\varepsilon}_{n+1_{I}}^{r}=\Delta \bar{\varepsilon}_{n+1}^{r},
$$

or again $r=0$ with

$$
\boldsymbol{r}=\boldsymbol{C}_{M}^{S}:\left[\Delta \boldsymbol{\varepsilon}_{n+1_{I}}^{r}-\frac{1}{f_{M}} \mathcal{S}^{-1}:\left(\Delta \varepsilon_{n+1_{I}}^{r}-\Delta \bar{\varepsilon}_{n+1}^{r}\right)\right]-\mathcal{C}_{I}^{S}: \Delta \boldsymbol{\varepsilon}_{n+1_{I}}^{r}
$$

In order to satisfy $r=0$, Eq. (B.9) is linearized as

$$
d r=\frac{\partial r}{\partial \varepsilon_{I}}: d \Delta \varepsilon_{I}^{r}+\frac{\partial r}{\partial \varepsilon_{M}}: d \Delta \varepsilon_{M}^{r}+\frac{\partial r}{\partial \bar{\varepsilon}}: d \Delta \bar{\varepsilon}^{r} .
$$


When solving $r=0$ at constant $\Delta \bar{\varepsilon}^{r}$, since $f_{M} \Delta \varepsilon_{n+1_{M}}^{r}+f_{I} \Delta \varepsilon_{n+1_{I}}^{r}$ is also a constant, the iteration process relies on $d r=J: d \varepsilon_{I}$ with

$$
\begin{aligned}
\boldsymbol{J} & =\frac{\partial \boldsymbol{r}}{\partial \boldsymbol{\varepsilon}_{I}}+\frac{\partial \boldsymbol{r}}{\partial \boldsymbol{\varepsilon}_{M}}: \frac{\partial \boldsymbol{\varepsilon}_{M}}{\partial \boldsymbol{\varepsilon}_{I}} \\
& =\boldsymbol{C}_{n+1_{M}}^{S}:\left[\boldsymbol{I}-\mathcal{S}^{-1}\right]-\boldsymbol{C}_{n+1_{I}}^{\mathcal{S}}-\frac{\partial \boldsymbol{C}_{n+1_{I}}^{S}}{\partial \varepsilon_{I}}: \Delta \boldsymbol{\varepsilon}_{n+1_{I}}^{r}-\frac{f_{I}}{f_{M}} \frac{\partial \boldsymbol{C}_{n+1_{M}}^{S}}{\partial \boldsymbol{\varepsilon}_{M}} \\
& :\left[\Delta \boldsymbol{\varepsilon}_{n+1_{I}}^{r}-\mathcal{S}^{-1}: \frac{\Delta \boldsymbol{\varepsilon}_{n+1_{I}}^{r}-\Delta \overline{\boldsymbol{\varepsilon}}_{n+1_{I}}^{r}}{f_{M}}\right]-\frac{f_{I}}{f_{M}} \boldsymbol{C}_{n+1_{M}}^{S} \otimes\left(\Delta \boldsymbol{\varepsilon}_{n+1_{I}}^{r}-\Delta \overline{\boldsymbol{\varepsilon}}_{n+1}^{r}\right) \\
& ::\left(\mathcal{S}^{-1} \otimes \mathcal{S}^{-1}\right):: \frac{\partial \mathcal{S}}{\partial \boldsymbol{\varepsilon}_{M}}-\frac{f_{I}}{f_{M}} \boldsymbol{C}_{n+1_{M}}^{S}: \mathcal{S}^{-1},
\end{aligned}
$$

where $\frac{\partial \mathcal{C}_{\alpha}^{S}}{\partial \boldsymbol{\varepsilon}_{\alpha}}$ results from Eq. (B.2).

When the residual-incremental-secant operator is used in the matrix phase, the derivative of the Eshelby tensor is given by, where we have omitted the subscript “M"::

$$
\frac{\partial \mathcal{S}}{\partial \Delta \varepsilon^{r}}=\frac{\partial \mathcal{S}}{\partial v} \otimes\left(\frac{\partial v}{\partial k^{S r}} \frac{\partial k^{S r}}{\partial \Delta \varepsilon^{r}}+\frac{\partial v}{\partial G^{S r}} \frac{\partial G^{S r}}{\partial \Delta \varepsilon^{r}}\right)
$$

with, using Eq. (60)

$$
\frac{\partial k^{S r}}{\partial \Delta \varepsilon^{r}}=0
$$

and

$$
\frac{\partial G^{S r}}{\partial \Delta \varepsilon^{r}}=\left[\frac{1}{6 G^{S r}\left(\Delta \varepsilon^{e q}\right)^{2}} \operatorname{dev}(\Delta \tau): C^{a l g}-\frac{2}{3} G^{S r} \frac{d e v\left(\Delta \varepsilon^{r}\right)}{\left(\Delta \varepsilon^{e q}\right)^{2}}\right] .
$$

When the zero-incremental-secant operator is used in the matrix phase, the derivative of the Eshelby tensor is given by, where we have omitted the subscript "M":

$$
\frac{\partial \mathcal{S}}{\partial \Delta \mathcal{E}^{r}}=\frac{\partial \mathcal{S}}{\partial v} \otimes\left(\frac{\partial v}{\partial k^{S 0}} \frac{\partial k^{S 0}}{\partial \Delta \boldsymbol{\varepsilon}^{r}}+\frac{\partial v}{\partial G^{S 0}} \frac{\partial G^{S 0}}{\partial \Delta \mathcal{\varepsilon}^{r}}\right)
$$

with using Eq. (64)

$$
\frac{\partial k^{S 0}}{\partial \Delta \varepsilon^{r}}=\frac{k^{e l}-k^{S 0}}{\operatorname{tr}\left(\Delta \varepsilon_{n+1_{\alpha}}^{r}\right)} \boldsymbol{I}
$$

and

$$
\frac{\partial G^{S 0}}{\partial \Delta \varepsilon^{r}}=\left[\frac{1}{6 G^{S 0}\left(\Delta \varepsilon^{e q}\right)^{2}} \operatorname{dev}(\Delta \tau): C^{a l g}-\frac{2}{3} G^{S 0} \frac{d e v\left(\Delta \varepsilon^{r}\right)}{\left(\Delta \varepsilon^{e q}\right)^{2}}\right]
$$


Once $r=0$ is satisfied, the effect on the strain increment in each phase of a variation $d \Delta \bar{\varepsilon}^{r}$ can directly be obtained by constraining $d r=0$, and Eq. (B.10) leads to $^{2}$

$$
0=\frac{\partial r}{\partial \varepsilon_{I}}: d \Delta \varepsilon_{I}^{r}+\frac{\partial r}{\partial \varepsilon_{M}}: d \Delta \varepsilon_{M}^{r}+\frac{\partial r}{\partial \bar{\varepsilon}}: d \Delta \bar{\varepsilon}^{r},
$$

or again

$$
\frac{\partial \varepsilon_{I}}{\partial \bar{\varepsilon}}=-J^{-1}: \frac{\partial r}{\partial \bar{\varepsilon}} .
$$

As under these circumstances $d \bar{\varepsilon}^{r}=f_{M} d \varepsilon_{M}^{r}+f_{I} d \varepsilon_{I}^{r}$, this last equation is completed by

$$
\frac{\partial \varepsilon_{M}}{\partial \bar{\varepsilon}}=\frac{1}{f_{M}}\left(\mathcal{I}-f_{I} \frac{\partial \varepsilon_{I}}{\partial \bar{\varepsilon}}\right) .
$$

\footnotetext{
${ }^{2}$ Note that the derivative with respect to $\Delta \varepsilon_{\alpha}^{r}$ has the same expression as the derivative with respect to $\varepsilon_{\alpha}$.
} 